CMSCs
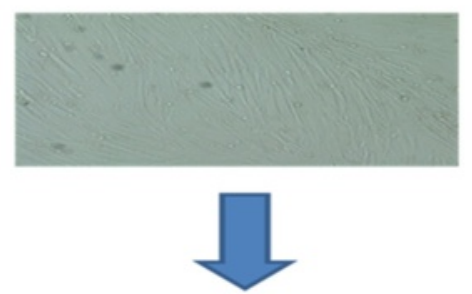

Myelo supportive Cytokines
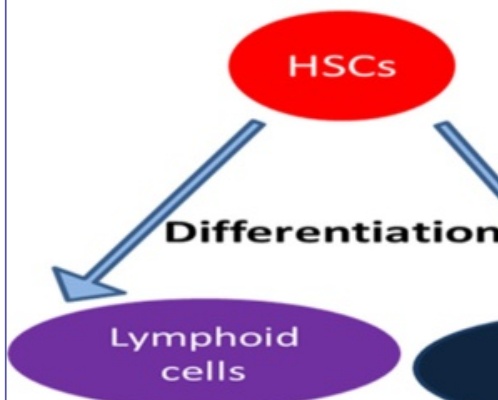

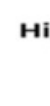

$$
\begin{aligned}
& \text { High Canonical WNT Signaling Pre osteoblastic potential }
\end{aligned}
$$

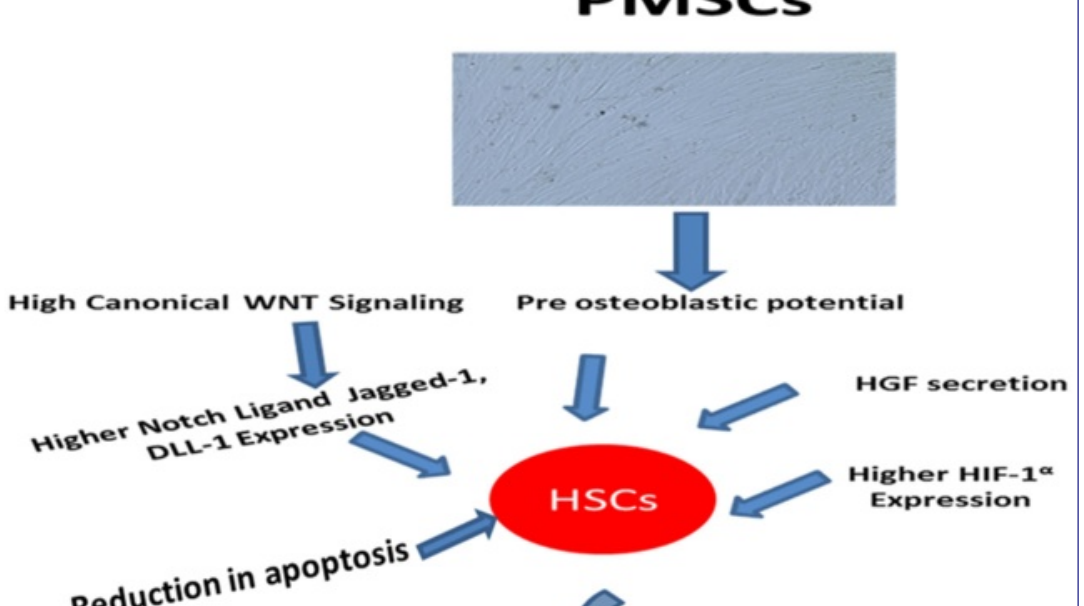

Higher Notch Ligand JagB
DLL-1 Expression

Reduction in apoptosis

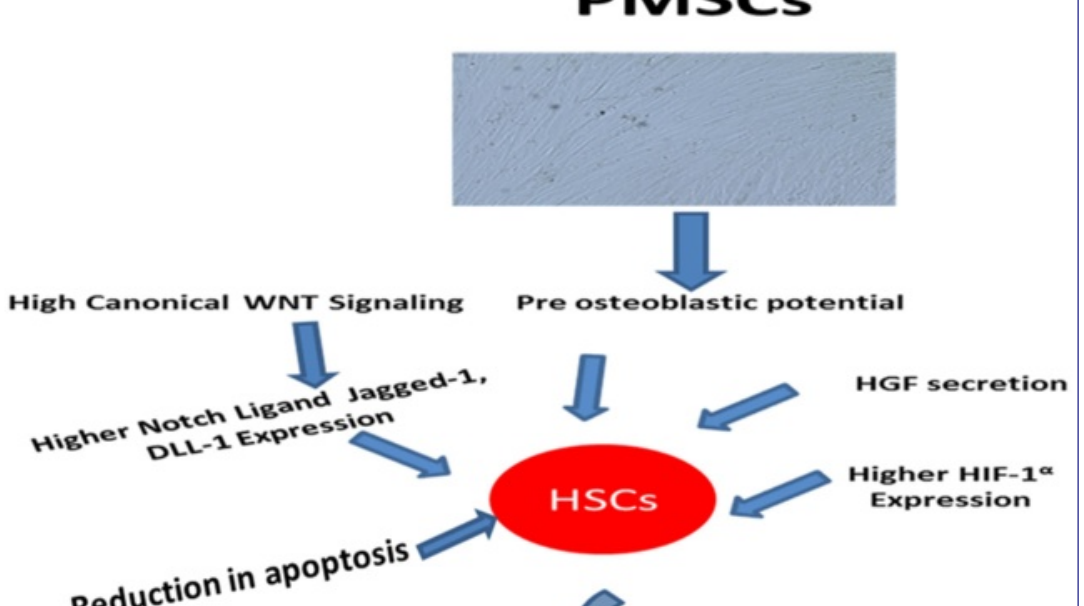

PMSCS

Self Renewal

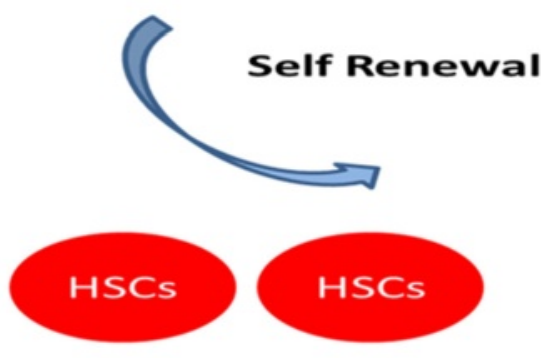

Myeloid

cells

Self Renewal

Differentiation

Self Renewal
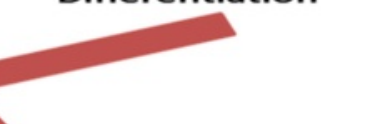

Differentiation

\title{
Differential ability of MSCs isolated from placenta and cord as feeders for supporting ex vivo expansion of umbilical cord blood derived CD34 ${ }^{+}$cells
}

Kadekar et al. 


\title{
Differential ability of MSCs isolated from placenta and cord as feeders for supporting ex vivo expansion of umbilical cord blood derived CD34 ${ }^{+}$cells
}

Darshana Kadekar, Vaijayanti Kale and Lalita Limaye*

\begin{abstract}
Introduction: Ex vivo expansion of umbilical cord blood (UCB) is attempted to increase cell numbers to overcome the limitation of cell dose. Presently, suspension cultures or feeder mediated co-cultures are performed for expansion of hematopoietic stem cells (HSCs). Mesenchymal stem cells (MSCs) have proved to be efficient feeders for the maintenance of HSCs. Here, we have established MSCs-HSCs co-culture system with MSCs isolated from less invasive and ethically acceptable sources like umbilical cord tissue (C-MSCs) and placenta (P-MSCs). MSCs derived from these tissues are often compared with bone marrow derived MSCs (BM-MSCs) which are considered as a gold standard. However, so far none of the studies have directly compared C-MSCs with P-MSCs as feeders for ex vivo expansion of HSCs. Thus, we for the first time performed a systematic comparison of hematopoietic supportive capability of C and P-MSCs using paired samples.
\end{abstract}

Methods: UCB-derived CD34 $4^{+}$cells were isolated and co-cultured on irradiated C and P-MSCs for 10 days. C-MSCs and P-MSCs were isolated from the same donor. The cultures comprised of serum-free medium supplemented with $25 \mathrm{ng} / \mathrm{ml}$ each of SCF, TPO, Flt-3 L and IL-6. After 10 days cells were collected and analyzed for phenotype and functionality.

Results: C-MSCs and P-MSCs were found to be morphologically and phenotypically similar but exhibited differential ability to support ex vivo hematopoiesis. Cells expanded on P-MSCs showed higher percentage of primitive cells $\left(\mathrm{CD} 34^{+} \mathrm{CD} 38^{-}\right)$, CFU (Colony forming unit) content and LTC-IC (Long term culture initiating cells) ability. CD34 ${ }^{+}$cells expanded on P-MSCs also exhibited better in vitro adhesion to fibronectin and migration towards SDF-1a and enhanced NOD/SCID repopulation ability, as compared to those grown on C-MSCs. P-MSCs were found to be closer to BM-MSCs in their ability to expand HSCs. P-MSCs supported expansion of functionally superior HSCs by virtue of reduction in apoptosis of primitive HSCs, higher Wht and Notch activity, HGF secretion and cell-cell contact. On the other hand, C-MSCs facilitated expansion of progenitors $\left(\mathrm{CD} 34^{+} \mathrm{CD} 38^{+}\right)$and differentiated $\left(\mathrm{CD} 34^{-} \mathrm{CD} 38^{+}\right)$cells by secretion of IL1-a, $\beta$, MCP-2, 3 and MIP-3a.

Conclusions: P-MSCs were found to be better feeders for ex vivo maintenance of primitive HSCs with higher engraftment potential than the cells expanded with C-MSCs as feeders.

Keywords: Ex vivo HSC expansion, Cord mesenchymal stem cells, Placental mesenchymal stem cells

\footnotetext{
* Correspondence: Islimaye@nccs.res.in

Stem Cell Laboratory, National Centre for Cell Science, University of Pune

Campus, Ganeshkhind, Pune 411007, Maharashtra, India
}

(c) 2015 Kadekar et al. Open Access This article is distributed under the terms of the Creative Commons Attribution 4.0 International License (http://creativecommons.org/licenses/by/4.0/), which permits unrestricted use, distribution, and reproduction in any medium, provided you give appropriate credit to the original author(s) and the source, provide a link to the Creative Commons license, and indicate if changes were made. The Creative Commons Public Domain Dedication waiver (http://creativecommons.org/publicdomain/zero/1.0/) applies to the data made available in this article, unless otherwise stated. 


\section{Introduction}

Over the past two decades, umbilical cord blood (UCB) has developed into an attractive and alternative source of hematopoietic stem cells (HSCs) both in clinics and in research. However, insufficient numbers of HSCs in the UCB limits its application in adults as an allogeneic source of HSCs for the transplantation [1]. The limited cell dose can be enhanced either by performing double CB transplantation (DCBT) or by ex vivo expansion of UCB. Ex vivo expansion stands out to be the most convenient option over the DCBT since, in the latter, there is successful engraftment of only a single $\mathrm{CB}$ unit with an elevated risk of graft versus host disease [1-4].

Currently, HSCs are expanded in the presence of a combination of cytokines [4-6]. However, under in vivo conditions, HSCs are reliant upon the cytokines and also on the varied components from their niche, such as mesenchymal stem cells (MSCs), endothelial cells, osteoblasts, etc., and extra cellular matrix for their maintenance and differentiation [7]. This emphasizes the need for an optimized culture system which closely resembles the in vivo niche and supports the growth of HSCs in vitro. Various cell types from the niche are used for the expansion of HSCs, but MSCs in particular are found to be efficient in sustaining the ex vivo expansion of HSCs [8-11].

Although BM remains the main source of MSCs, here we have established MSCs-HSCs co-cultures with MSCs isolated from non-invasive sources, such as umbilical cord and placenta [12]. It is reported that MSCs obtained from these sources are morphologically and phenotypically comparable with BM-MSCs $[13,14]$. CMSCs may be situated as an alternative to BM-MSCs in the field of HSCs transplantation as opposed to P-MSCs which are primarily explored as a valuable source for cell replacement therapies. Despite intensive investigation, to the best of our knowledge no report has directly compared the HSCs supportive function of these two stromal populations. We report here a unique observation that $\mathrm{C}$-MSCs and P-MSCs have differential propensities for the maintenance of HSCs. To decipher the basis of the differential ability of these feeders to support the ex vivo maintenance and propagation of HSCs we isolated CMSCs and P-MSCs from the same donor.

We demonstrate here that P-MSCs make better feeders than C-MSCs, and were found to possess similar potential as BM-MSCs for expansion of primitive UCB HSCs. Conversely, expansion mediated by $\mathrm{C}$-MSCs was primarily dependent on the pro-inflammatory cytokines secreted by them yielding differentiated cells. We also report that the differences observed are a reflection of not only the prominent activation of Wnt and Notch signals but also of improved survival signals from P-MSCs as opposed to C-MSCs. Based on all the findings, we conclude that
P-MSCs are the most suitable feeders for the ex vivo maintenance of functional HSCs.

\section{Methods}

Ethical approvals for human samples and animal experiments

UCB samples, placenta, and cord were collected from local hospitals after obtaining informed consent with the compliance of the institutional review board (IEC-Institutional ethical committee -NCCS and IC-SCR -Institutional Committee for Stem Cell Research, NCCS) according to the Declaration of Helsinki. Consenting procedures were also approved by the NCCS-IC-SCR- Institutional Committee for Stem Cell Research, NCCS.

Protocols for animal experiments were approved and in accordance with the IAEC-NCCS Institutional Animal Ethical Committee-NCCS/CPCSEA- Committee for the Purpose of Control and Supervision of Experiments on Animals. (Approval number: IACUC-Institutional Animal Care and Use Committee, EAF-Experimental Animal Facility/2004/B-71(III)). Throughout the experiments animals were under the supervision of trained personnel.

\section{Collection of umbilical cord blood and isolation of CD34+ cells}

Cord blood samples were collected from local hospitals after obtaining informed consent with the compliance of the institutional review board (IEC-Institutional ethical committee and IC-SCRT -Institutional Committee for Stem Cell Research, NCCS) according to the Declaration of Helsinki. Consenting procedures were also approved by the IC-SCR- Institutional Committee for Stem Cell Research, NCCS. Mononuclear cells were isolated by the ficoll hypaque density gradient method (density $1.077 \mathrm{~g} / \mathrm{ml}$, Sigma Aldrich, St. Louis MO, USA). CD34+ cells were isolated by the positive selection method using Dynal beads according to the manufacturer's instructions (Dynabeads M-450 CD34; Dynal, ASA, Oslo, Norway).

\section{Isolation of mesenchymal stem cells from cord tissue and placenta}

Umbilical cord and placenta were collected from full term deliveries after obtaining informed consent in accordance with the IEC-Institutional ethical committee and ICSCRT- Institutional Committee for Stem Cell Research, NCCS. The umbilical cord and a central piece from the placenta were used to isolate the mesenchymal stem cells. The detailed procedure is explained in Ref [15]. Cells between passages three to six were used for the experiments after carrying out the phenotypic characterization of the MSCs. Phenotypic characterization and differentiation to osteoblasts and adipocytes and chondrocytes was carried out as explained in [15]. 
Isolation of mesenchymal stem cells from bone marrow BM-MSCs were a kind gift from Dr.Mohan Wani, NCCS (Pune). BM-MSCs were cultured in RPMI + $20 \%$ Mesen FBS (GIBCO, Grand Island, NY, USA). Phenotypic characterization and differentiation to osteoblasts and adipocytes was carried out as explained earlier [15]. Chondrogenic differentiation was carried out using a StemPro Chondrogenesis kit (Invitrogen, Grand Island, NY, USA) according to the manufacturer's instructions.

\section{Establishment of co-cultures of MSCs and HSCs in serum-free medium}

MSCs were seeded in 24-well plates (BD Falcon, San Jose, CA, USA) at a cell density of $10^{4}$ cells/well and were grown until confluence. The monolayers were then irradiated at 8,000 rads and $5 \times 10^{4}-1 \times 10^{5} \mathrm{UCB}$ CD $34^{+}$cells in Stem Pro Serum Free medium (GIBCO) were seeded onto the monolayers for contact cultures. For non-contact cultures, $10^{4}$ cells were seeded on $3 \mu \mathrm{m}$ cell culture inserts (Millicell, Millipore, San Diego, USA). The cocultures were incubated for ten days in the presence of a growth factor cocktail containing IL-6, SCF, TPO, and Flt-3-L. All growth factors (Peprotech Inc., Rocky Hill, NJ, USA) were used at a final concentration of $25 \mathrm{ng} /$ $\mathrm{ml}$. After ten days both the non-adherent and adherent fractions were collected and studied for various parameters such as phenotypic characterization, in vitro functionality assessment, and in vivo engraftment studies in NOD/SCID mice. Assessment of these parameters was carried out using independent cord blood samples.

\section{Flow cytometry analysis}

The expanded cells were subjected to phenotypic characterization using a panel of anti-bodies. For the detection of intracellular proteins the cells were permeabilized using a BD fixperm kit (BD Pharmingen, San Jose, CA, USA) and the staining was done according to the manufacturer's instructions. Isotype matched antibodies were used as controls. The fluorescently labeled cells were acquired on FACS Canto II and Aria (BD, San Jose, CA, USA) and data were analyzed by FACS DIVA - version 5.0 The details of the antibodies used are as follows: annexin V FITC, CD34 APC/PE, CD38 FITC, Bcl-2 PE, Bax FITC, CD33 FITC/PE, CD19 APC CD3 FITC, CD61 APC, CD45 PE/PE-CY-7 murine CD45.1 Pacific blue (BD Pharmingen) and CD133 PE (Milteny Biotech, Colonge, Germany).

\section{Colony-forming unit (CFU) assay}

Expanded cells $\left(2 \times 10^{4}\right)$ were cultured in $1 \%$ methylcellulose (Sigma Aldrich) with a combination of the growth factors: SCF $20 \mathrm{ng} / \mathrm{mL}$, GM-CSF $2 \mathrm{ng} / \mathrm{mL}$, IL-3 $4 \mathrm{ng} / \mathrm{mL}$, and Epo $2 \mathrm{U} / \mathrm{mL}$. The plates were incubated under humidified conditions at $37{ }^{\circ} \mathrm{C}$, in $5 \% \mathrm{CO} 2$ for
14 days. Based on the differential morphology of the colonies they were scored as blast-forming unit erythroid (BFU-E), granulocyte-monocyte (GM), granulocyteerythroid-monocyte-megakaryocyte (GEMM), and megakaryocytes (MK).

\section{Long-term culture initiating cell (LTC-IC) assay}

M210B4 (murine stromal cell line, ATCC) cells was grown on collagen-coated wells of 24-well plates to confluency. The plates were gamma-irradiated (8,000 rads) and $1 \times 10^{5}$ expanded cells were seeded on feeder layers in human myelocult medium (Stem Cell Technology, Vancouver, Canada) supplemented with hydrocortisone $\left(10^{-6} \mathrm{M}\right)$. The cultures were maintained for four to eight weeks with weekly demi-defoliation. LTC-IC activity was assessed by the CFU assay.

\section{In vitro migration towards SDF1a}

Expanded cells $\left(1 \times 10^{5}\right)$ were seeded in the upper chamber of a transwell migration set up ( $8 \mu \mathrm{BD}$ Falcon). A total of $100 \mathrm{ng}$ of SDF $1 \alpha$ was added to the lower wells containing $600 \mathrm{ml}$ of medium (IMDM + $2 \%$ FCS). One set was run without the addition of SDF1 $\alpha$ as a control to assess spontaneous migration. The cells were allowed to migrate for five $\mathrm{h}$ at $37{ }^{\circ} \mathrm{C}$. The migrated cells were collected, manually counted and a graph of total migrated cells in both sets was plotted after subtracting the number of spontaneously migrated cells from the total number of migrated cells. Further, the migrated fraction was analyzed to check the $\mathrm{CD} 34^{+} \mathrm{CD} 38^{-}$content.

\section{Cell - matrix adhesion assay}

Adhesive capacities of the expanded cells were evaluated by determining their adhesion to fibronectin. The CD34+ cells were sort purified from the expanded cells and $10^{5}$ cells were seeded on fibronectin-coated 96-well plates. The plates were incubated for $35 \mathrm{~min}\left(37^{\circ} \mathrm{C}, 5 \% \mathrm{CO} 2\right)$. Unbound cells were gently washed off and the adhered cells were fixed using $4 \%$ paraformaldehyde (ten min, ambient temperature) and stained using crystal violet $(0.1 \%$ in $70 \%$ methanol). The plates were washed three times using sterile distilled water to remove the excess stain and the cells were then lysed with Triton X 100 (30 min, ambient temperature) to release the crystal violet dye. The absorbance was measured at $550 \mathrm{~nm}$. A higher absorbance corresponded with more adhesion.

\section{NOD/SCID repopulation assay}

The NOD/LtSZ-scid/scid mice (Jackson Laboratory, Bar Harbor, ME, USA) were bred in the animal facility of NCCS. All the protocols were approved and in accordance with IAEC-NCCS Institutional animal ethical committee -NCCS/CPCSEA- Committee for the Purpose of Control and Supervision of Experiments on Animals.(Approval 
number: IACUC-Institutional Animal Care and Use Committee, EAF-Experimental Animal Facility/2004/B-71(III)). Throughout the experiments animals were under the supervision of trained personnel. Mice at four to six weeks of age were exposed to a sub-lethal dose of 300 rads total body irradiation from a ${ }^{60} \mathrm{Co}$ source (Gammachamber5000, BRIT, Navi Mumbai, India). Expanded cells $\left(10^{6}\right)$ from C- and P-MSCs sets were then infused through the tail vein into the sub-lethally irradiated mice $(n=5-10)$. Short-term and long-term engraftment was assessed in bone marrow and spleen at the 4th and 12th weeks after infusion by probing human $\mathrm{CD} 45^{+}$cells against the host murine CD45 background. The presence of greater than $0.1 \%$ in the total nucleated cells of the recipient was considered as a successful engraftment. The multi-lineage engraftment from the donor was determined using a panel of antibodies against the following markers: CD34 ${ }^{+}$(stem cell marker), CD33 (myeloid cells), CD3, CD19, and CD56 (lymphoid cells). The antibodies were purchased from BD Pharmingen. The respective isotype antibodies (Beckton Dickinson, San Jose, CA, USA) were included as control. For staining of peripheral blood, $100 \mu \mathrm{l}$ of blood was incubated with the antibodies, further RBCs were lysed with lysis buffer (BD) and washed twice with PBS containing $0.1 \%$ BSA. A minimum of 1,00,000 events were acquired on FACS Canto II (BD).

\section{Apoptosis detection by annexin V/PI assay}

After ten days of expansion, cells were harvested and $10^{5}$ cells were taken for annexinV/PI staining. Briefly, the cells were first washed with PBS and re-suspended in $1 \times$ binding buffer. A total of $5 \mu \mathrm{L}$ of annexin V FITC (BD Biosciences) was added and incubated at room temperature for $20 \mathrm{~min}$. Cells were again washed with 1 $x$ binding buffer to remove the excess of antibody. Prior to acquisition on flow cytometer, $0.5 \mu \mathrm{g}$ of propidium iodide was added to each tube and then the cells were analyzed by flow cytometry.

\section{Wnt and Notch signaling in MSCs-HSCs co-cultures by western blot}

P- and C-MSCs were irradiated and cell lysates were prepared after $96 \mathrm{~h}$ of irradiation in RIPA buffer. For expanded cells, CD34+ cells were sorted after expansion and the cell lysate was prepared in RIPA buffer. A total of $30 \mu \mathrm{g}$ of protein was loaded on $10 \%$ SDS-PAGE. Protein samples were then transferred to a PVDF membrane and overnight blocking with $5 \%$ BSA in TBST was carried out. For detection, the panel of antibodies listed below (Cell Signaling Technology) was used. For detection, a chemiluminescent based detection kit from Cell Signaling Technology was used. Densitometric analysis was performed using Image J software and each protein was normalized to the respective $\beta$-actin levels.
Primary antibodies were: $\beta$-catenin (D10AB) XP rabbit $\mathrm{mAB}$, phospho $\beta$-catenin (Ser33/37/Thr41), phospho $\beta$ catenin (Ser552), TCF-1 (C63D9) LEF1 (C12A5), Dll-1, jagged-1, notch1(D6F11), cleaved notch 1 (NICD V1744) (D3BB), Bmi-1 and Hes. Secondary antibodies were: anti-rabbit IgG HRP-linked $\mathrm{Ab}$ and anti-Mouse IgG HRP-linked Ab.

\section{Cytokine array}

Cytokines and chemokines present in the CMs were measured using the human Cytokine Array C5 (AAHCYT-5-8) following the manufacturer's instructions (Ray Biotech, Norcross GA, USA). Densitometry analyses were performed using Image J software. All values were normalized according to the mean intensity of positive and negative controls. Fold change was calculated according to the manufacturer's instructions.

\section{Statistical analysis}

The statistical differences between groups were analyzed by one way repeated measure analysis of variance using the software SIGMA STAT (Jandel Scientific Corporation, San Rafael, CA, USA) for all the experiments. The values were plotted as mean \pm standard deviation Probability values ${ }^{*} \mathrm{p} \leq 0.05$, ${ }^{* *} \mathrm{p} \leq 0.01$, and ${ }^{* * *} \mathrm{p} \leq 0.001$ were considered statistically significant.

\section{Results}

C- and P-MSCs share similar morphology, phenotype and differentiation potential towards osteoblasts, adipocytes and chondrocytes

First, we isolated MSCs from cord (C-MSCs) and placenta (P-MSCs). C- and P-MSCs had similar fibroblastic morphologya (Fig. 1a). C- and P-MSCs showed similar marker expression of molecules such as CD44, CD73, CD105 and CD90, and were found to be negative for CD34, CD45, CD14, CD19, CD11b and HLA-DR which is in accordance with the International Society for Cellular Therapy criteria for defining MSCs [16] (Fig. 1b). Furthermore, we evaluated their multi-lineage differentiation potential by inducing them to differentiate into osteoblasts, adipocytes, and chondrocytes. We found that C- and P-MSCs could be differentiated into all three lineages (Fig. 1c).

\section{P-MSCs were superior to C-MSCs and closer to BM-MSCs} for the expansion of primitive HSCs

We next compared C-MSCs and P-MSCs for their ability to support hematopoiesis. Initially we used C-MSCs and P-MSCs isolated from different donors. As we observed striking differences in the expansion efficiency of the two MSCs (data not shown), expansion was attempted using $\mathrm{C}$ - and P-MSCs from paired samples.

Equal numbers of the CD $34^{+}$cells from a single cord blood unit were seeded separately on pre-established 


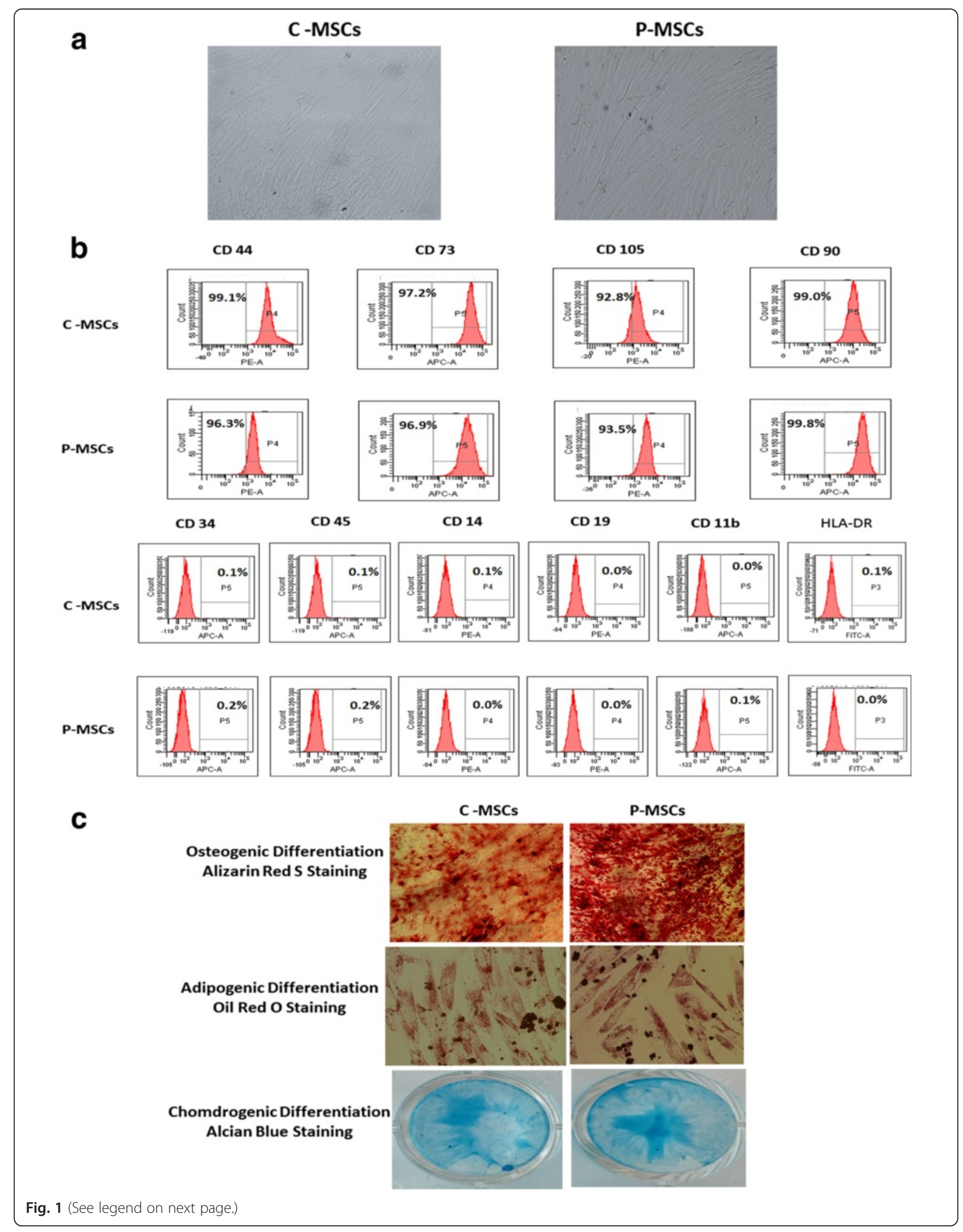


(See figure on previous page.)

Fig. 1 C- and P-MSCs displayed similar morphology and immuno-phenotype. a Fibroblastic morphology exhibited by C- and P-MSCs as seen under phase contrast microscope (10X). b Histogram profile of representative samples of C- and P-MSCs exhibiting expression of markers such as CD44, CD90, CD73, and CD105. No expression was found for CD45, CD34, CD14, CD19, CD11b, and HLA-DR. c Upper panel represents osteogenic differentiation of MSCs by staining with alizarin red S. Middle panel is for adipogenic differentiation confirmed after lipid droplets stained by oil red o. Chondrogenic differentiation of C- and P-MSCs confirmed with Alcian blue stain. C-MSCs cord-derived mesenchymal stem cells, P-MSCs placenta-derived mesenchymal stem cells

irradiated C- and P-MSCs obtained from the same donor and were cultured for ten days. CD $34^{+}$cells expanded on P-MSCs (P-MSCs:CD34 ${ }^{+}$co-cultures) displayed a significant (two-fold) increase in total nucleated cell (TNC) yield, a 2.5 -fold increase in immature hematopoietic progenitors $\left(\mathrm{CD}_{3} 4^{+} \mathrm{CD} 38^{-}\right)$and a higher proportion of the most primitive $\mathrm{CD} 133^{+}$cells compared to those expanded with C-MSCs (C-MSCs:CD34 ${ }^{+}$co-cultures) (Fig. 2a). Figure $2 \mathrm{~b}$ upper panel is a dot plot representation which shows an increase in the $\mathrm{CD} 34^{+} \mathrm{CD} 38^{-}$cells $(39.5 \%)$ in the P-MSCs:CD34 ${ }^{+}$co-cultures while C-MSCs:CD34 ${ }^{+}$cocultures harbored committed cells CD $34^{+} \mathrm{CD} 38^{+}$(34.9\%). The histogram of CD133 expression on $\mathrm{CD} 45^{+} \mathrm{CD} 34^{+}$ cells from a representative sample is shown in Fig. $2 \mathrm{~b}$ (lower panel).

To further validate our observation we compared the hematopoietic supportive function of C-MSCs and PMSCs with that of the gold standard, i.e., BM-MSCs. BM-MSCs were thus used as a positive control. BMMSCs used in these experiments were first characterized morphologically, phenotypically, and by studying their differentiation ability towards three lineages, i.e., osteo, adipo, and chondro. They were found to satisfy the criteria laid down by ISCT to be identified as MSCs (see Additional file 1: Figure S1a and b). When the three types of MSCs were compared for their ability to expand UCB HSCs, it was observed that P-MSCs though not superior to BM-MSCs, were closer to them. On the contrary, C-MSCs were found to be the least efficient in expanding UCB HSCs. This was evident from higher expansion of the $\mathrm{CD}^{+} 4^{+} \mathrm{CD}^{-} 8^{-}$sub-population and the most primitive $\mathrm{CD} 133^{+}$fraction (see Additional file 1: Figure S1c), as well as from their higher clonogenicity (see Additional file 1: Figure S1d) when the $\mathrm{CD}^{+}{ }^{+}$cells were expanded on BM-MSCs and P-MSCs as compared to C-MSCs.

\section{P-MSCs expanded HSCs without hindering their quiescence state}

Since P-MSCs had resulted in the massive expansion of HSCs, we next checked if the expansion was caused at the expense of exhausting the stem cell pool. A significantly higher percentage of $\mathrm{CD}_{3} 4^{+}$cells were in the G0/G1 phase in P-MSCs:CD34 ${ }^{+}$co-cultures as compared to the CMSCs set (Fig. 2c). The left panel of Fig. 2c shows a representative FACS profile of PI based cell cycle wherein an increase in the cycling HSCs (as represented by the S/ G2M phase of the cell cycle) is seen in C-MSCs:CD34 ${ }^{+}$ co-cultures. Figure $2 \mathrm{~d}$ depicts a representative FACS profile of Hoechst and PyroninY based resolution of the G0/G1 phase of the cell cycle, where P-MSCs:CD34 ${ }^{+}$cocultures showed that $37 \%$ of the $\mathrm{CD} 4^{+}$cells were in the G0 phase as opposed to $17 \%$ in C-MSCs:CD34 ${ }^{+}$cocultures. This observation clearly indicates that P-MSCs supported proliferation of $\mathrm{CD} 34^{+}$cells without compromising its quiescence state.

\section{$\mathrm{CD} 4^{+}$cells expanded with P-MSCs exhibited augmented in vitro functional attributes}

Owing to the fact that C- and P-MSCs co-cultures presented differences in the phenotype of the expanded population, we next determined their functionality. PMSCs:CD34 ${ }^{+}$co-cultures showed greater clonogenicity as evaluated by CFU assay and manifested significantly higher BFU-E (four-fold), CFU-GEMM (1.6-fold), CFUMK (1.5-fold), and total CFU (1.5-fold) as compared to C-MSCs:CD34+ co-cultures (Fig. 3a).

We further analyzed the self-renewal capacity of expanded cells by the LTC-IC assay. Both co-cultures revealed the formation of differential colonies in the LTC-IC assay. However, P-MSCs:CD34 ${ }^{+}$co-cultures harbored significantly higher total LTC-IC units as compared to C-MSCs:CD34 ${ }^{+}$co-cultures (Fig. 3b).

Trans-well migration assay is an in vitro method to ensure chemotaxis of expanded cells towards SDF- $1 \alpha$ which is an important step for their successful lodgment in the BM. Cells expanded on P-MSCs showed a significantly higher number of migratory cells than cells expanded on C-MSCs (Fig. 3c). Upon analysis of the migrated fraction it was observed that the migrated fraction of $\mathrm{P}$ MSCs:CD34 ${ }^{+}$co-cultures had approximately two-fold more primitive $\mathrm{CD} 34^{+} \mathrm{CD} 38^{-}$cells than cells co-cultured with C-MSCs (Fig. 3d). This augmented chemotactic response was attributed to the significantly higher percentage of $\mathrm{CD} 34^{+} \mathrm{CXCR}-4^{+}$population in the P-MSCs:CD34 ${ }^{+}$ co-cultures than in the C-MSCs:CD34+ co-cultures (Fig. 3e). A representative FACS profile is shown as an inset in Fig. 3e.

After transplantation, HSCs establish themselves in the microenvironment via adhesive interactions with cellular and ECM components. We next checked their ability to adhere to fibronectin in vitro. Cells expanded 


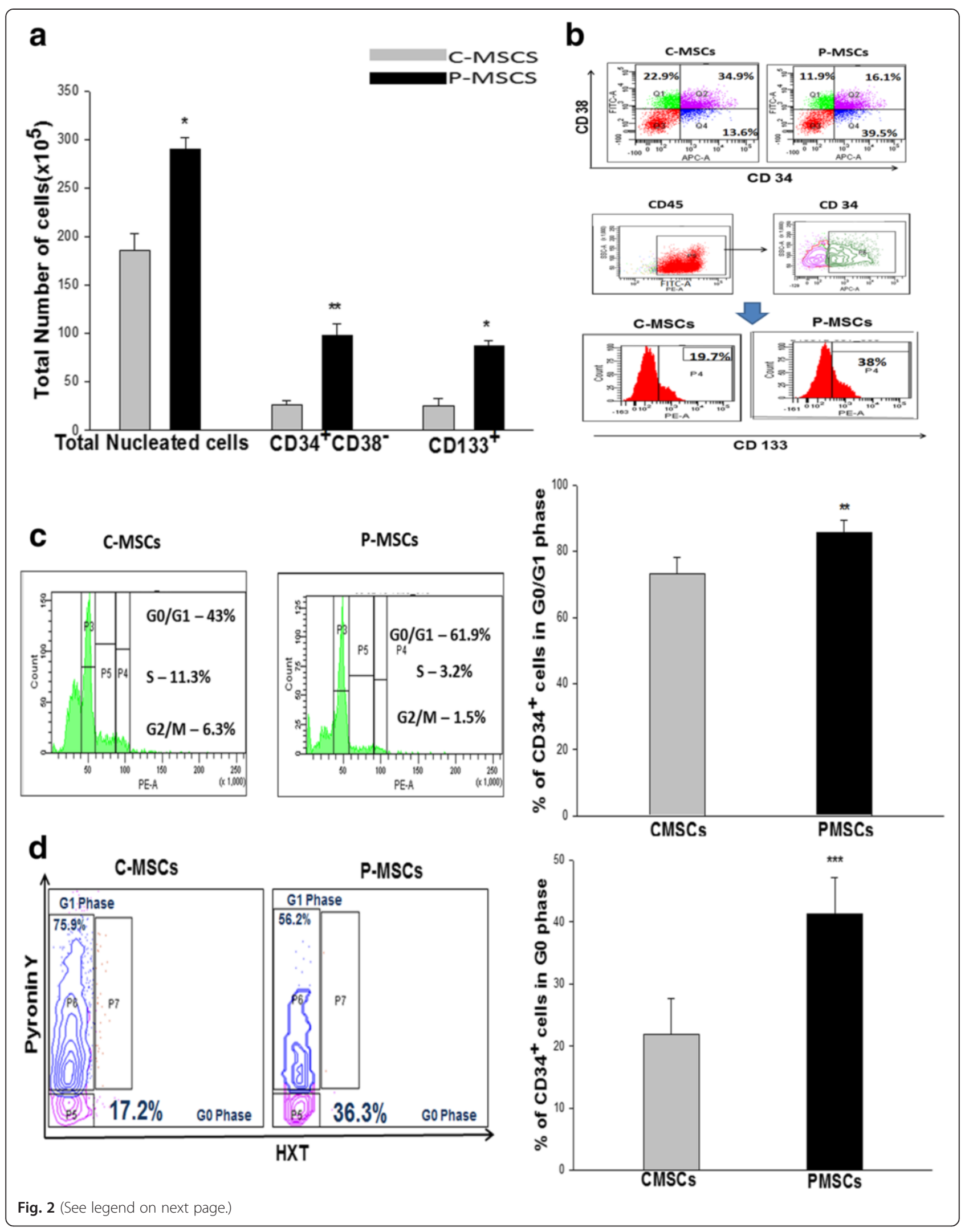


(See figure on previous page.)

Fig. 2 P-MSCs supported robust expansion of CD34 ${ }^{+}$cells without deterring their quiescence state. a Co-culturing of CD34 ${ }^{+}$cells with P-MSCs harbored a significantly higher TNC yield, CD $34^{+} \mathrm{CD} 38^{-}$and $\mathrm{CD} 133^{+}$cells than C-MSCs as feeders. $\mathbf{b}$ Upper panel- A representative FACS dot plot showing higher $\mathrm{CD} 34^{+} \mathrm{CD} 38^{-}$cells with P-MSCs as feeders. Lower panel- A representative FACS histogram depicting total $\mathrm{CD} 34^{+} \mathrm{CD} 133^{+}$cells in the co-culture C- and P MSCs as feeders. c Cell cycle analysis demonstrated an increase of CD34 ${ }^{+}$cells at G0/G1 phase with P-MSCs as feeders and higher numbers of cycling CD34+ cells in cultures comprised of C-MSCs as feeders. d FACS dot plot profile of Hoechst and PyroninY based cell cycle analysis showing P-MSCs as feeders had a higher percentage of CD34 ${ }^{+}$cells in G0 (quiescence) stage in the co-cultures with P-MSCs than C-MSCs. Data are represented as mean \pm standard deviation from three independent sets of experiments. ${ }^{*} p \leq 0.05,{ }^{*} p \leq 0.01$, and ${ }^{* * *} p \leq 0.001$ P-MSCS placenta-derived mesenchymal stem cells, C-MSCs cord-derived mesenchymal stem cells, FACS fluorescence-activated cell sorting

on P-MSCs exhibited significantly superior adhesion to fibronectin as evident from the higher number of crystal violet stained cells seen attached to fibronectin (Fig. $3 \mathrm{f}$ inset), as compared to the cells expanded on C-MSCs. The attachment was also quantified by lysing the cells and measuring the absorbance at $550 \mathrm{~nm}$ (Fig. 3f).

\section{Co-culturing of CD34 ${ }^{+}$cells with P-MSCs exhibited enhanced SCID repopulation ability Primary engraftment}

Expanded cells $\left(10^{6}\right)$ were infused into sub-lethally irradiated NOD/SCID mice and four weeks after infusion short term engraftment in the BM was assessed by probing human CD45 cells against the host murine CD45 background. An irradiated mouse infused with PBS was used as a negative control. Cells expanded on P-MSCs showed significantly higher chimerism in the $\mathrm{BM}$ and spleen of NOD/SCID mice than those expanded on C-MSCs (Fig. 4a and b). Representative FACS dot plots, revealing a higher percentage of $\mathrm{HuCD} 45^{+}$cells in the BM of NOD/SCID mice infused with cells expanded on P-MSCs, are shown in Fig. 4c. The multi-lineage engraftment was confirmed by examining the donor-derived CD34 population, myeloid cells (CD33), and lymphoid cells (CD3, CD19, and CD56) in the $\mathrm{BM}$ of recipient mice. No predisposition for any specific lineage was observed (Fig. 4d). Further donorderived committed progenitors were evaluated by an in vitro colony formation assay on MBM using human specific growth factors. A significantly higher CFU content was found in the BM of mice that received cells from PMSCs:CD34+ co-cultures after four weeks (Fig. 4e).

To ensure the long term engraftment potential, the presence of human CD $45^{+}$cells was also evaluated in the MBM after 12 weeks. Approximately three- and 1.5-fold higher chimerism was detected in BM and spleen, respectively, with the cells from P-MSCs:CD $34^{+}$co-cultures than with the cells from C-MSCs:CD34 ${ }^{+}$co-cultures (Fig. 4f and g). A FACS profile depicting the same is shown in Fig. 4h. It should be noted that the percentage of huCD45 increased in the MBM after 12 weeks owing to the in vivo proliferation of the infused cells. The engrafted cells were able to undergo multi-lineage differentiation as shown in
Additional file 2: Figure S2a. Significantly higher human CFC content was obtained with P-MSCs:CD34 ${ }^{+}$cocultures as quantified and depicted in Additional file 2: Figure S2b.

\section{Secondary transplantation}

A serial transplantation assay was carried out to ensure the in vivo self-renewal of co-cultured HSCs. For that, BM cells from the primary recipient were harvested 12 weeks post transplantation and $10^{5}$ sort-purified huCD45 were re-infused into sub-lethally irradiated secondary recipient NOD/SCID mice. Four weeks post transplantation, cells from the P-MSCs:CD34 ${ }^{+}$co-cultures gave rise to 2.5-fold and two-fold higher chimerism in BM and spleen, respectively, than with C-MSCs as feeders (Fig. 4i and j). A representative FACS profile is depicted in Fig. 4k. The engrafted cells retained their multi-lineage differentiation potential as depicted in Additional file 2: Figure S2c which was further confirmed by quantitating human derived CFC in MBM by the CFU assay, as shown in Additional file 2: Figure S2d.

\section{P-MSCs supported survival of $\mathrm{CD}_{3}{ }^{+}$cells via up-regulation of anti-apoptotic $\mathrm{BCl}-2$ protein}

We next determined if the above observed improvement was due to a difference in the survival of the cells expanded with the two feeders. A significant reduction was observed in the apoptosis in the P-MSCs:CD34 $4^{+}$cocultures as opposed to that with C-MSCs:CD34 ${ }^{+}$cocultures. A significantly higher percentage of annexin $\mathrm{V}^{-} \mathrm{PI}$ - cells (viable cells) were observed in the total (1.2-fold) and $\mathrm{CD}_{3} 4^{+}$(1.5-fold) compartment in the P-MSCs:CD34 ${ }^{+}$ co-cultures than in the C-MSCs:CD $34^{+} \mathrm{C}$ o-cultures (Fig. 5a). Figure 5b depicts the FACS profile of a representative sample.

In order to study the mechanism behind this differential survival, the expression of $\mathrm{BCl}-2$ and $\mathrm{Bax}$ was evaluated. It was found that P-MSCs had significantly upregulated $\mathrm{BCl}-2$ expression in the primitive $\left(\mathrm{CD} 34^{+} \mathrm{CD} 38\right.$ $\left.{ }^{-}\right)(57.34 \pm 3.45 \%)$ and progenitor $\left(\mathrm{CD} 34^{+} \mathrm{CD} 38^{+}\right)(\sim 95 \%)$ populations, while relatively low expression was observed in the totally differentiated cells $\left(\mathrm{CD} 34^{-} \mathrm{CD} 38^{+}\right)(52.32 \pm$ $4.23 \%)$. In sharp contrast, C-MSCs showed the highest expression of $\mathrm{BCl}-2$ in totally differentiated cells ( $\geq 95 \%)$ 


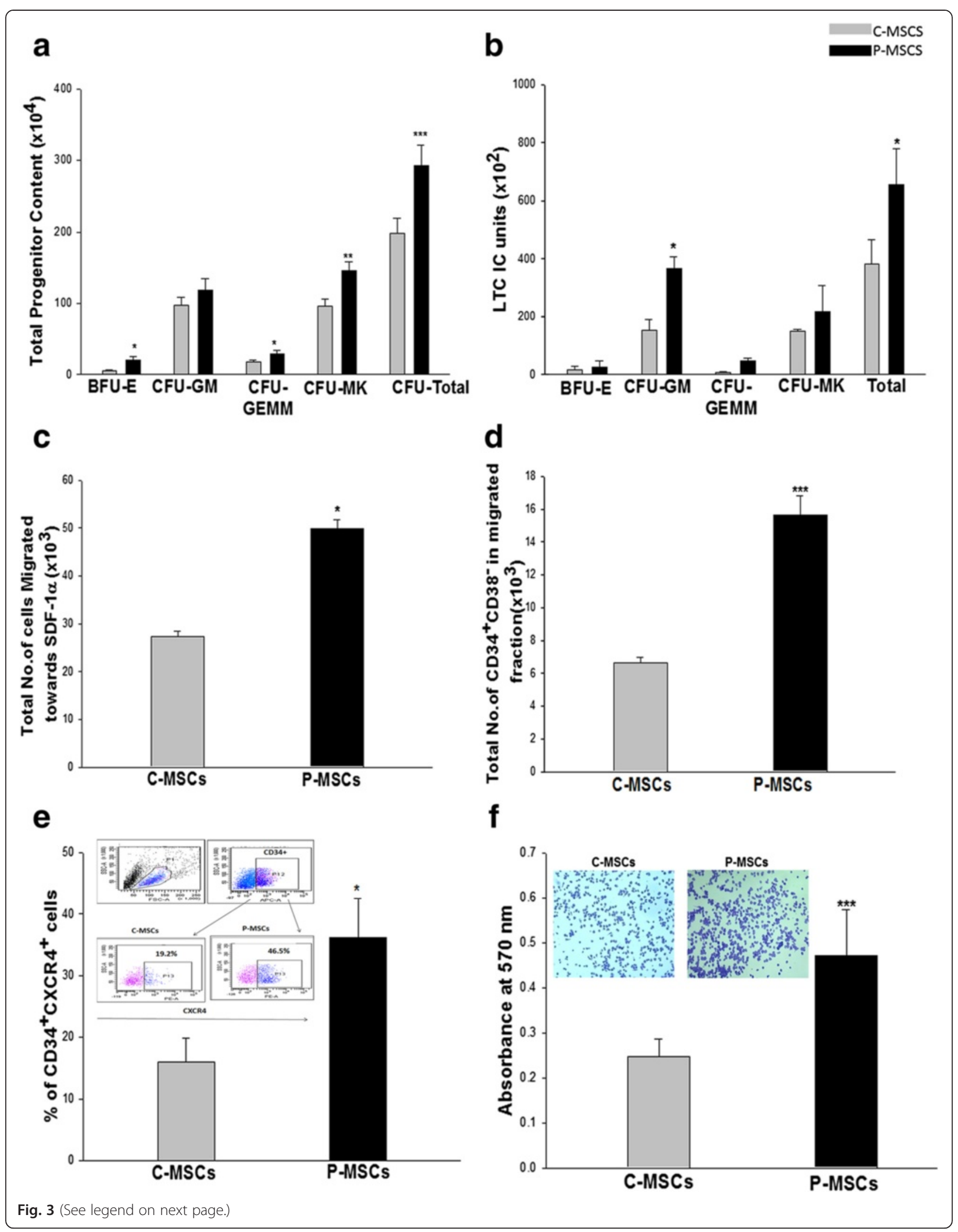


(See figure on previous page.)

Fig. $3 \mathrm{CD}^{+} 4^{+}$cells expanded on P-MSCs have augmented in vitro functionality of the expanded cells. a P-MSCs:CD34+ Co-cultures displayed higher blast-forming unit erythroid (BFU-E), granulocyte-monocyte (GM), granulocyte-erythroid-monocyte megakaryocyte (GEMM), and megakaryocytes (MK) colonies when compared with C-MSCs as feeders. b P-MSCs as feeders maintained significantly higher LTC-IC units than C-MSCs. c Trans well migration assay displayed significantly augmented migration of the expanded cells towards SDF-1a in the P-MSCs set as compared to the C-MSCs set. $\mathbf{d}$ Migrated fraction from the co-culture with P-MSCs revealed significantly higher numbers of primitive CD34 ${ }^{+} \mathrm{CD} 38^{-}$cells. e Superior migratory response might be attributed to the higher percentage of CD34 ${ }^{+} \mathrm{CXCR}-4^{+}$cells in co-Cultures with P-MSCs as feeders. $\mathbf{f}$ Quantitation of the attachment to the fibronectin was carried out after lysing the adhered cells and measuring color intensity at $570 \mathrm{~nm}$. The inset shows sort-purified CD34 ${ }^{+}$expanded on P-MSCs adhered to fibronectin in higher numbers. Data are represented as mean \pm standard deviation from three independent sets of experiments. ${ }^{*} p \leq 0.05$, ${ }^{* *} p \leq 0.01$, and ${ }^{* * *} p \leq 0.001$. P-MSCs placenta-derived mesenchymal stem cells, C-MSCs cord-derived mesenchymal stem cells, LTC-IC long term culture initiating cell

and much lower expression in the primitive $(17.98 \pm$ $3.76 \%)$ and progenitors $(79.12 \pm 1.45 \%)$ populations. Conversely, P-MSCs:CD34 ${ }^{+}$co-cultures demonstrated significantly lower levels $(\leq 60 \%)$ of Bax protein in the primitive population $(5.25 \pm 1.25 \%)$, progenitors $(41.06 \pm$ $3.69 \%)$, and totally differentiated cells (59.76 $\pm 5.45 \%)$, while the Bax expression was higher in the primitive population (38.54 $\pm 5.85 \%$ ), progenitors $(94.23 \pm 2.15 \%)$, and totally differentiated cells $(90.56 \pm 2.55 \%)$ with CMSCs as feeders (Fig. 5c). It should be noted that the percentage of $\mathrm{Bax}^{+} \mathrm{CD} 34^{+} \mathrm{CD} 38^{-}$was negligible in the cells expanded on P-MSCs.

We have earlier reported that the addition of antiapoptotic agents resulted in increased expansion efficiency of suspension cultures of UCB CD34 ${ }^{+}$cells [17]. Since higher levels of apoptosis were detected in the $\mathrm{C}$ MSCs:CD34 ${ }^{+}$co-cultures, we checked if this system could be made equivalent to the P-MSCs co-cultures by the addition of anti-apoptotic molecules. Addition of the pancaspase inhibitor zVADfmk (500nM) and the calpain-1 inhibitor zLLYfmk $(15 \mu \mathrm{M})$ significantly increased the yield of TNC (see Additional file 3: Figure S3a) and primitive cells (see Additional file 3: Figure S3b) compared to those without inhibitors. Also, an improvement was seen in the functionality of the cells expanded with inhibitors as assessed by the CFU assay (see Additional file 3: Figure $\mathrm{S} 3 \mathrm{c}$ ), in vitro migration towards SDF1 $\alpha$ (see Additional file 3: Figure S3d) and in vitro adhesion to fibronectin (see Additional file 3: Figure S3e).

\section{Physical interaction between P-MSCs and CD34+ cells is indispensable for the beneficial effect of P-MSCs}

Next we checked if the modulatory effect of P-MSCs was contact or cytokines dependent. A significant reduction ( $\geq 50 \%$ ) was observed in the TNC yield (see Additional file 4: Figure S4a), the total CD34+ content, and primitive content (see Additional file 4: Figure S4b) in non-contact cultures as compared to contact cultures of P-MSCs with $\mathrm{CD}^{+} 4^{+}$cells. However, noncontact co-cultures of C-MSCs showed a marginal reduction in cell yield (see Additional file 3: Figure S3a). The viability of the cells in the non-contact cultures of P-MSCs was greatly compromised, as evident from a significant reduction in the percentage of total live (48.62 \%) as well as CD34 ${ }^{+}$cells (38.67\%) (see Additional file 4: Figure S4c). On the contrary, no change in the viability of non-contact C-MSCs culture was observed (data not shown). Thus, physical contact with the P-MSCs is a key for the maintenance of HSCs.

To identify the molecular basis of these interactions, we carried out a series of blocking experiments. Out of the myriad of interacting molecules, we chose to focus on integrin ( $\alpha 4 \beta 1 /$ VCAM- 1 and $\alpha 5 \beta 1$ /fibronectin) and SDF$1 \alpha /$ CXCR- 4 since they are involved in providing survival signals to HSCs in the niche. Blocking of VLA-4 $(\alpha 4 \beta 1)$, VLA-5( $\alpha 5 \beta 1)$, and CXCR-4 on CD34 ${ }^{+}$cells was done singly or in combination with the blocking antibodies. The unblocked/blocked cells were then co-cultured with respective stroma for $96 \mathrm{hrs}$ and the proliferation was assessed by MTT. Individual blocking of the integrins had minimal effect on the survival of $\mathrm{CD} 4^{+}$cells in the cocultures with C-MSCs and P-MSCs (see Additional file 4: Figure S4d,e). Blocking with individual or combinations of antibodies had no effect on the proliferation (see Additional file 4: Figure S4d), clonogenic ability (see Additional file 4: Figure S4f) and survival (see Additional file 4: Figure S4h) of the cells expanded on C-MSCs. In P-MSCs:CD $34^{+}$co-cultures, however, all the above parameters were compromised, particularly upon blocking integrins in combination with CXCR-4 (see Additional file 4: Figure S4e, g.i).

The results clearly indicate that P-MSCs exert their effect partly via integrin and SDF- $1 \alpha$ mediated axis.

\section{Enhanced expansion on P-MSCs can be attributed to the functional differences between the two feeders}

With P- and C-MSCs having exhibited differences in the hematopoiesis supportive function, we further investigated the indigenous differences between these two stromal populations. P-MSCs showed a significantly higher rate of proliferation than C-MSCs (Fig. 6a). P-MSCs retained significantly higher clonogenecity even at low cell count $\left(10^{3}\right)$ cells as compared to C-MSCs (Fig. 6b and inset). C- and P-MSCs could be differentiated into osteoblasts, adipocytes, and chondrocytes. However, qualitatively and quantitatively P-MSCs displayed greater 


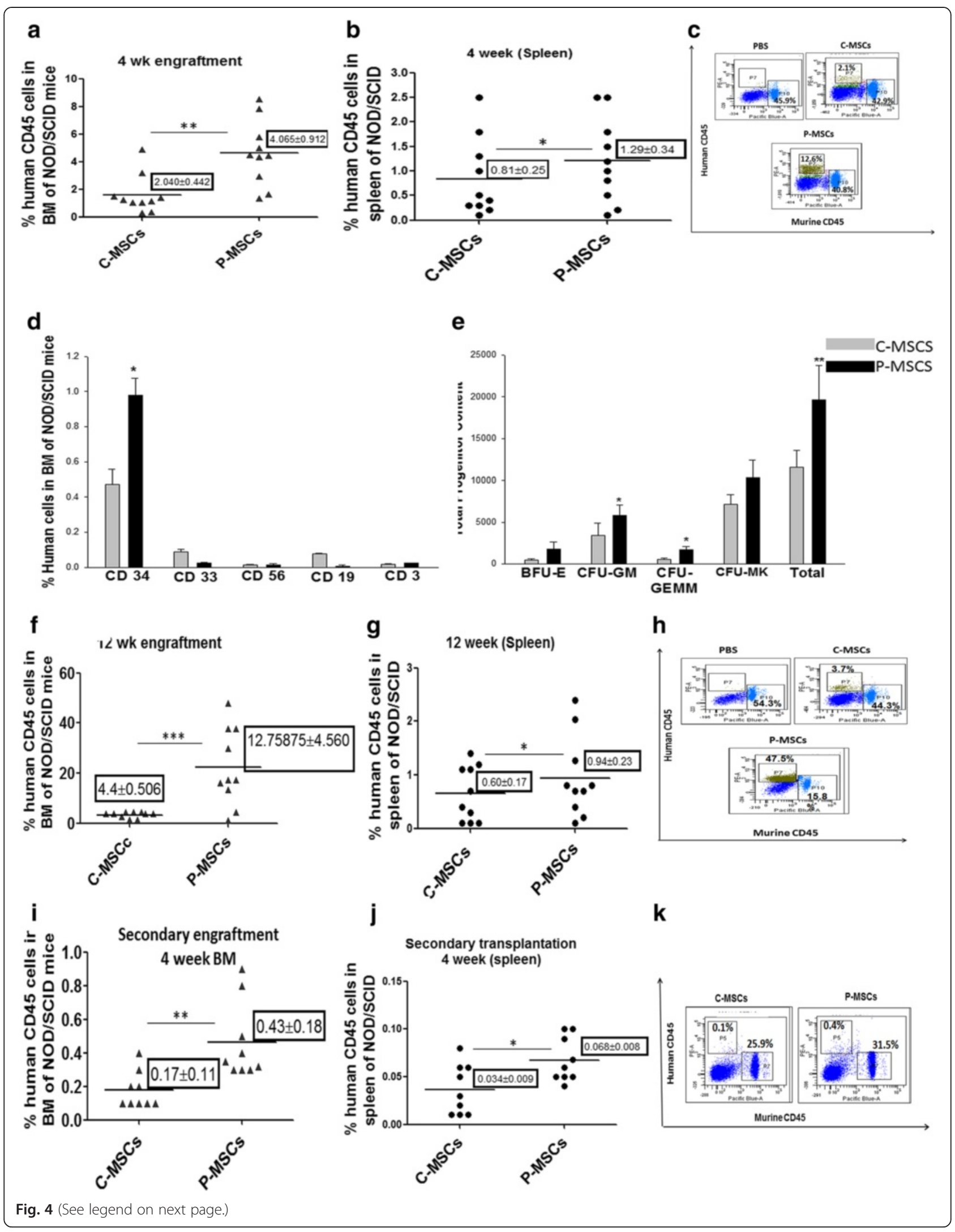


(See figure on previous page.)

Fig. 4 P-MSCS imparts significantly higher SCID repopulating ability on expanded CD34 $4^{+}$cells than C-MSCs. a,b At four weeks post transplantation (short term engraftment) the bone marrow(a) and spleen(b) of the mice receiving cells expanded on P-MSCs showed a significantly higher percentage of human $C D 45^{+}$cells. Each dot in the graph represents an individual animal. c Representative FACS dot plots showing a higher percentage of human CD45 ${ }^{+}$cells in the BM of NOD/SCID mice infused with cells expanded on P-MSCs on the background of murine CD45 cells. $\mathbf{d}$ Donor derived7 multilineage engraftment was seen in the BM of NOD/SCID mice for P- and C-MSCs expanded sets as determined by CD34 (stem cells), CD33 (myeloid cells), CD56(NK cells), CD19 (B cells), and CD3 (T cells). e Donor-derived committed progenitors were higher in the BM of mice receiving cells expanded on P-MSCs than on C-MSCs as evaluated by performing an in vitro colony formation assay on MBM using human specific growth factors. $\mathbf{f}, \mathbf{g}$ Long term engraftment (12 weeks) indicated significantly higher chimerism (\% human CD45) was detected in the BM ( $f$ ) and spleen ( $\mathrm{g}$ ) of NOD/SCID mice receiving cells from P-MSCs co-cultures. $\mathbf{h}$ Representative FACS dot plots showing higher percentage of human CD45 cells in BM of NOD/SCID mice infused with cells expanded on P-MSCs in the murine CD $45^{+}$background after 12 weeks. $\mathbf{i}$, $\mathbf{j}$ Serial transplantation assay showed a significantly higher percentage of human $C D 45^{+}$in $B M$ (i) and spleen (j), the secondary recipient receiving cells from mice infused with cells expanded on P-MSCs. $\mathbf{k}$ Representative FACS dot plots showing a higher percentage of human $\mathrm{CD}^{2} 5^{+}$cells in the BM of NOD/SCID mice infused with cells from primary recipient. Data are represented as mean \pm standard deviation from ten different mice. $(n=10){ }^{*} p \leq 0.05$,

${ }^{* *} p \leq 0.01$, and ${ }^{* * *} p \leq 0.001$. P-MSCS placenta-derived mesenchymal stem cells, C-MSCs cord-derived mesenchymal stem cells, FACS

fluorescence-activated cell sorting, $B M$ bone marrow

osteogenic potential as compared to C-MSCs (Fig. 6c left panel). To corroborate these findings, we further checked the levels of RUNX-2 in P-MSCs and C-MSCs. It was found that the expression of RUNX-2 was three-fold higher in P-MSCs (Fig. 6d).

Some of the attributes of P-MSCs, such as predisposition to the osteoblastic lineage and higher RUNX-2 expression, were found to be similar to MSCs found in the endosteum, where hypoxia leads to the stabilization of HIF- $1 \alpha$ which is a key regulator of hematopoiesis. Therefore, we checked if there was any difference in HIF-1 $\alpha$ level in both MSC populations. P-MSCs showed significantly higher normoxic stabilization of HIF- $1 \alpha$ than C-MSCs. Fig. 6e shows the confocal images of the nuclear stabilization of HIF-1 $\alpha$ in C- and P-MSCs under normoxic conditions. No difference was observed for hypoxic stabilization of HIF- $1 \alpha$ in C and P-MSCs (data not shown).

The balance between differentiation and maintenance of the stem cells is regulated by complex molecular mechanisms involving soluble factors and cell-cell interactions. Therefore, we compared the secretory profile of C-MSCs and P-MSCs using a semi-quantitative cytokine array (Fig. 6g). The majority of the cytokines secreted by $\mathrm{C}$-MSCs were of a pro-inflammatory nature with a more than two-fold up-regulation of IL-1 $\alpha$, IL-1 $\beta$, MCP-2, MCP-3, and MIP- $3 \alpha$ observed, whereas the quantities of these cytokines were negligible in the CM of P-MSCs except for HGF (Fig. $6 f$ and h). The graph represents a quantitative analysis of two independent paired samples.

\section{P-MSCs displayed higher Wnt activation resulting in improved Notch signaling in the expanded HSCs}

To elucidate the molecular mechanism behind the better hematopoiesis observed in P-MSCs:CD34 ${ }^{+}$co-cultures, we evaluated the levels of the Wnt and Notch signaling pathways, which are linked to self-renewal and maintenance of HSCs. We observed a 1.5-fold higher expression of $\beta$-catenin, an effector of in irradiated P-MSCs than in irradiated C-MSCs (Fig. 7a). Further, phosphorylation at the ser-33/37/Thr41 residue which is linked to degradation of $\beta$-catenin mediated by GSK-3 $\beta$ was found to be reduced in the P-MSCs. The level of activator phosphorylation at residue ser-552 by AKT/PKA was also found to be 1.3-fold higher in P-MSCs. A downstream target of the canonical $\beta$-catenin pathway, the levels of TCF were found to be 1.5 -fold higher in P-MSCs stromal cells. Further, promoters of jagged- 1 and $\delta$-like one (dll- 1 ), also shown to be targets of $\beta$-catenin, were found to be upregulated in P-MSCs (Fig. 7a). Therefore, we investigated the corresponding activation of notch signaling in the HSCs expanded in the co-culture. Figure 7b shows significantly higher expression of total Notch 1 and cleaved Notch 1 (NICD form) in the CD $34^{+}$cells expanded on PMSCs (1.5-fold). The downstream targets of Notch, Bmi-1 and Hes-1 were also found to be up-regulated 2.5-fold and 1.2-fold, respectively, in the P-MSCs:CD34 ${ }^{+}$coculture (Fig 7c). Collectively, these results indicate that higher levels of Wnt/ $\beta$-catenin signals in the P-MSCs resulted in the activation of Notch signaling in the CD $34^{+}$ cells.

\section{Discussion}

Ex vivo expansion of UCB HSCs is attempted to increase the cell dose for HSCs transplantation in adult patients $[1,4,5]$. HSCs are either expanded in suspension cultures or in support with the different cell types from the hematopoietic niche $[6,8-10]$. Among the different cell types used for expansion of HSCs, MSCs have been found to be the most appropriate feeders [4]. Although $\mathrm{BM}$ remains the chief source of MSCs, obtaining this source involves an invasive procedure, thus posing a risk to the donor. The quality of MSCs is also influenced by the age of donors. On the contrary, the fact that MSCs from neonatal tissues can be isolated with ease, with no harm to the donor, and their primitive nature indisputably poses an attractive alternative to BM-MSCs. Thus, perinatal tissues may be ideal sources for cell therapy 


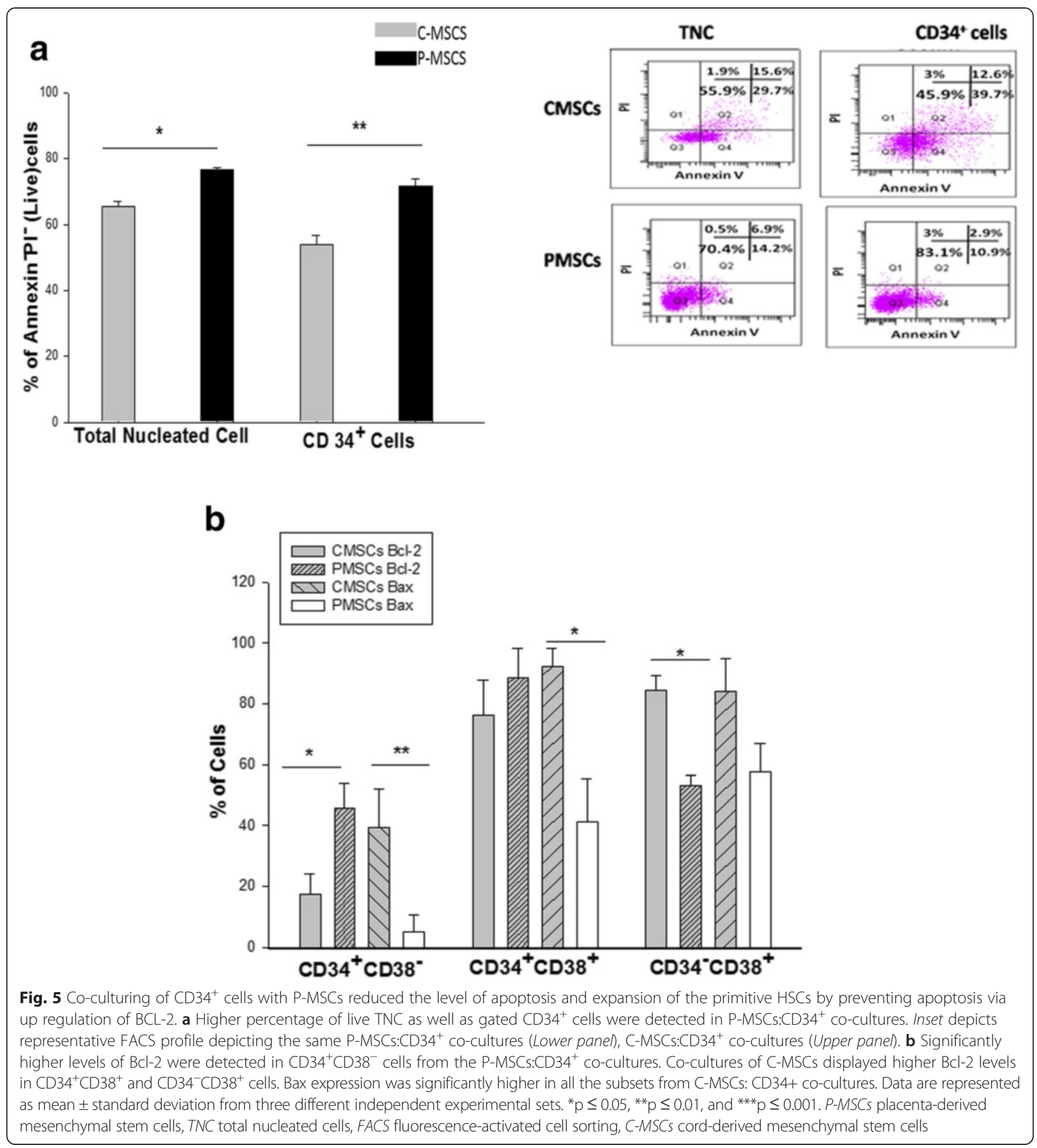

[13]. C- and P-MSCs are being extensively studied with reference to their immune modulatory functions and trans-differentiation potential and have proven to be better than BM-MSCs [14]. C-MSCs are further seen as a promising alternative to $\mathrm{BM}-\mathrm{MSCs}$ as feeders for expanding HSCs $[18,19]$. However, the potential of PMSCs as feeders for HSCs expansion is probably underestimated while it is being explored as a valuable source for cell replacement therapies. Despite extensive comparison of MSCs derived from these tissues with the BM-MSCs, to date none of these studies have directly compared the hematopoietic supportive function of CMSCs with that of P-MSCs. We found striking differences in their ability to serve as feeders, in that P-MSCs were found to be better feeders than C-MSCs for the support of ex vivo hematopoiesis. Furthermore, our observation with 


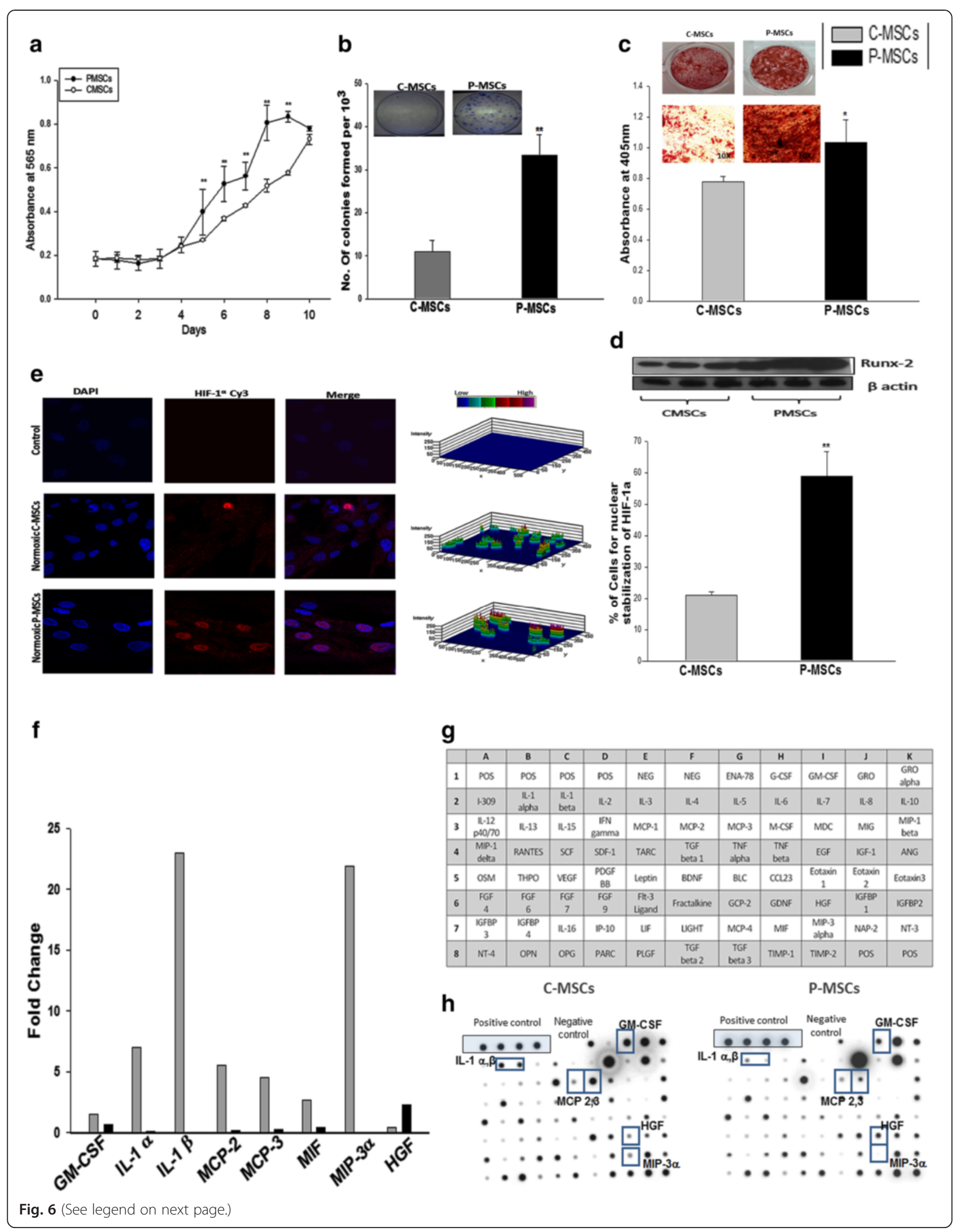


(See figure on previous page.)

Fig. 6 C-MSCs and P-MSCs differ in the functional attributes relevant to expansion of HSCs. a P-MSCs had higher rate of proliferation than C-MSCs as determined by MTT assay. b P-MSCs exhibited higher clonogenecity than C-MSCs even at lowest cell concentration. Inset depicts the representative CFU-F colonies stained with crystal violet. c P-MSCs exhibits superior differentiation to osteoblasts depicted by the calcium deposits stained with alizarin red S (Left panel). Right panel shows quantitative analysis after extraction of the dye and its quantitation at $405 \mathrm{~nm}$. $\mathbf{d}$ Superior osteoblastic differentiation may be due to the pre-osteoblastic nature of P-MSCs by virtue of higher RUNX-2 expression. e Higher normoxic stabilization of HIF-1a in the nucleus of the P-MSCs than C-MSCs. Left panel shows 3D representations of expression of the HIF-1a as 3-D histograms. Percentage of the positive nuclei from ten random fields/slide of three independent experiments is represented in the graph. $\mathbf{f}$ Fold change in the cytokines expression by C-MSCs and P-MSC, respectively. Data represented as mean value of two independent paired samples. $\mathbf{g}$ C-MSCs had a distinctive secretion profile mainly of pro-inflammatory cytokines as checked by membrane-based cytokine array. $\mathbf{h}$ Represents the hybridization results for a representative sample. Data are represented as mean \pm standard deviation from three different independent experimental sets. ${ }^{*} p \leq 0.05,{ }^{* *} p \leq 0.01$, and ${ }^{* * *} p \leq 0.001$. P-MSCs placenta-derived mesenchymal stem cells, C-MSCs cord-derived mesenchymal stem cells, HSCs hematopoietic stem cells, CFU-F colony forming unit-fibroblasts

the BM-MSCs as positive control additionally strengthens the inference that P-MSCs are equally as competent as BM-MSCs for supporting HSC expansion as opposed to C-MSCs. However, since our primary aim here was to compare C- and P-MSCs, the detailed mechanism underlying the difference in the ability to support hematopoiesis was studied with respect to C- and P-MSCs only and not with BM-MSCs.

C-MSCs and P-MSCs were found to be morphologically and phenotypically similar but it was observed that P-MSCs could be cultured over 11-15 passages, as opposed to C-MSCs that could be maintained for only seven to eight passages (data not shown). P-MSCs were found to be sturdier as they sustained the transition from FBS-containing medium to the xenofree medium consisting of cord blood derived plasma better than CMSCs [15]. These advantages make P-MSCs undoubtedly better and more convenient to maintain in cultures than C-MSCs. The superiority of P-MSCs for supporting the expansion of functionally superior and transplantable HSCs was established from the results of an array of in vitro and in vivo assays. P-MSCs promoted better expansion in terms of primitive progenitor content, CFU and LTC assays with improved adhesive and migratory properties. The results of in vivo assays, such as short/long term and secondary engraftment in the NOD/SCID mouse model, left little doubt that P-MSCs as feeders are more efficient at expansion of UCB HSCs than C-MSCs.

While optimizing the co-culture system, we opted to select irradiated MSCs as feeders for expansion of HSCs. In a clinical scenario, prior to HSC transplantation, patients usually undergo myeloablation in the form of radiation therapy. The transplanted HSCs then lodge on the irradiated stroma, proliferate, and establish hematopoiesis. Thus, irradiated stroma probably stimulates the HSCs. Taking this fact into consideration, along similar lines, we also compared the effect of unirradiated and irradiated MSCs on their capacity to support hematopoiesis and found that indeed irradiated MSCs had substantially increased efficiency to expand HSCs (data not shown). Available literature also supported our observation. Celebi et al. [20] have reported that irradiated MSCs partly mimic the endosteal niche thereby supporting better expansion of HSCs ex vivo. Furthermore, Walenda et al. [21] highlights the maintenance of primitive $\mathrm{CD} 34^{+} \mathrm{CD} 38^{-}$cells after culturing on irradiated bone marrow MSCs.

Next, in an attempt to seek the differences in the characteristics of the two feeders that regulate the hematopoietic supporting ability, it was found that P-MSCs robustly supported the expansion of primitive HSCs, as opposed to CMSCs, that supported the expansion of cells dominant in $\mathrm{CD}_{4} 4^{+} \mathrm{CD} 38^{+}$and $\mathrm{CD} 34^{-} \mathrm{CD} 38^{+}$progenitor cells. The observed expansion of different subsets in the two co-culture systems could be attributed to the differences in cytokines secreted by C- and P-MSCs. C-MSCs secrete a variety of the pro-inflammatory cytokines, such as IL- $1 \alpha, \beta$, GM-CSF and MCP-2, 3, driving differentiation of HSCs [22], thus validating the observed expansion of the progenitor population and decline in the primitive HSCs content in cocultures of C-MSCs. In sharp contrast, the P-MSCs secreted negligible levels of the above cytokines. Cytokines secreted by both the MSCs were similar to those reported in the literature for other MSCs, such as BM-MSCs, adipose MSCs, etc. [23, 24]. Still, we find striking differences in the levels of their secretion when we compared $\mathrm{C}$ - and P-MSCs. Further, Fong et al. [25] have reported better expansion of UCB CD34 ${ }^{+}$cells with the conditioned medium obtained from Wharton's jelly-derived MSCs (WJ-MSCs) than when co-cultured with MSCs. This clearly suggests that CM of WJ-MSCs plays a role in the expansion of HSCs even in the absence of the feeder cells themselves, which supports our observation about the cytokines secreted by C-MSCs and its effect on the expansion of committed progenitors.

The improved expansion in co-cultures of P-MSCs, however, cannot solely be attributed to the presence of HGF, whose levels were merely two-fold higher in PMSCs, as against the levels of C-MSCs cytokines. Our data on contact and non-contact cultures of P-MSCs convincingly demonstrate that P-MSCs exert a beneficial effect only upon physical contact with the HSCs. Thus, 


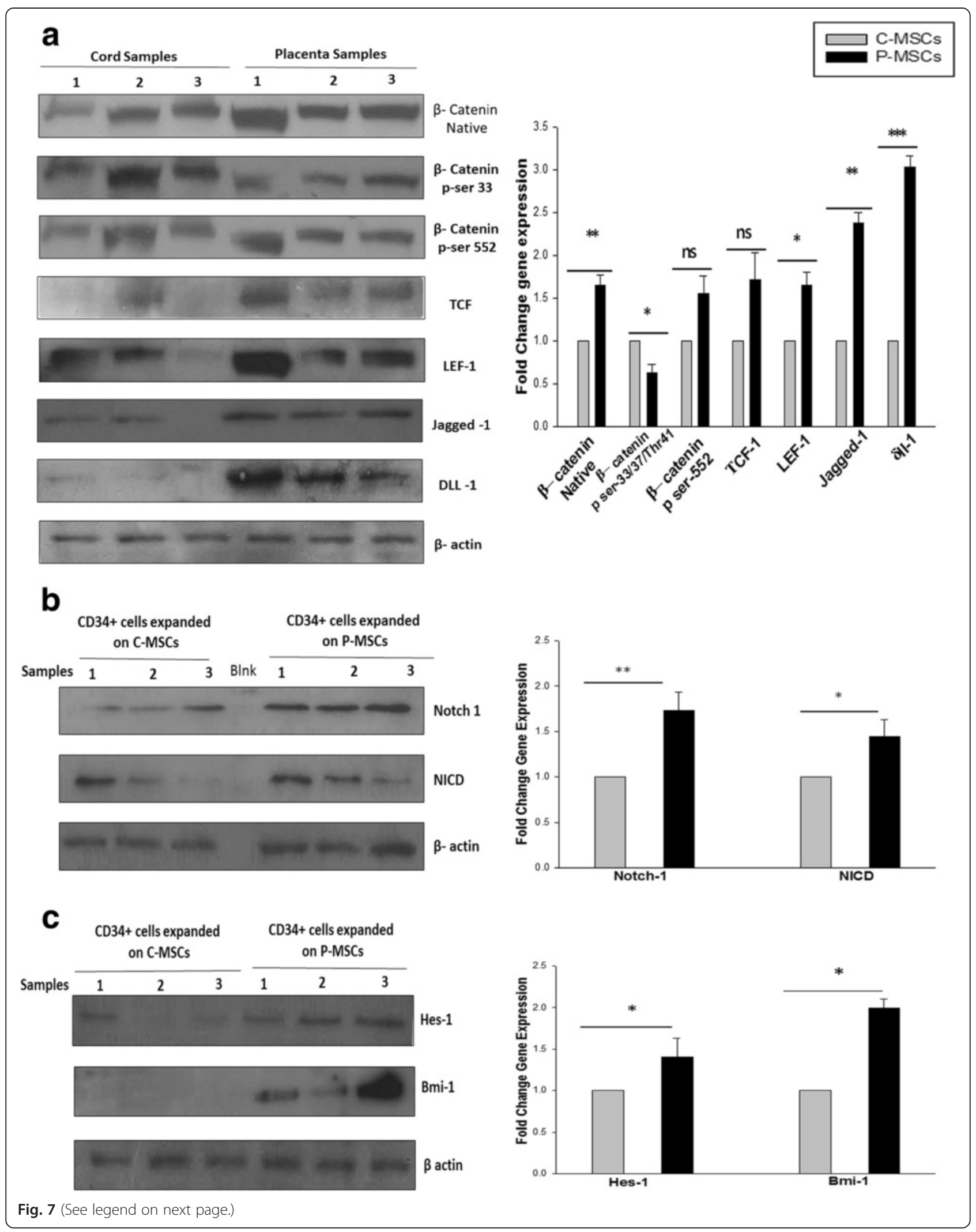


(See figure on previous page.)

Fig. 7 P-MSCs displayed higher Wnt activity leading to collateral increase into Notch signaling in the expanded CD34 ${ }^{+}$cells. a Higher expression of native $\beta$-catenin. Phosphorylated $\beta$-catenin (ser-552) was found in the P-MSCs. Higher expression of phosphorylated $\beta$-catenin (ser-33/34Thr41) in C-MSCs. The expression of downstream targets of Wnt, TCF, LEF-1, jagged-1 and $\delta$-like one (dll-1) was higher in P-MSCs than C-MSCs. The graph represents the fold change in the expression after normalization to $\beta$-actin. $\mathbf{b}$ Sort purified CD34 ${ }^{+}$cells from P-MSCs. Co-cultures showed significant up regulation of Notch I and intracellular domain of Notch (NICD). The quantitation of the same is represented as a graph. c The CD34 ${ }^{+}$cells expanded on the P-MSCs exhibited higher expression of Hes-1 and Bmi-1, downstream target of Notch, quantitatively represented as a graph. Data are represented as mean \pm standard deviation from three different samples. ${ }^{*} p \leq 0.05,{ }^{* *} p \leq 0.01$, and ${ }^{* * *} p \leq 0.001 . P$-MSCs placenta-derived mesenchymal stem cells, C-MSCs cord-derived mesenchymal stem cells

we investigated the contribution of other cellular factors that might be responsible for the expansion of primitive HSCs. One such crucial factor in deciding the HSC fate is cell-cell contact, as explained by Da Silva et al. [26]. At endosteum, HSCs interact with MSCs/osteoblast predominantly via (SDF-1/CXCR-4) or integrin mediated interactions [26, 27]. Likewise, Fonseca et al. [28] reported a significant increase in the primitive $\mathrm{CD}^{+} 4^{+} \mathrm{CD} 38^{-}$cells which were in close association with MSCs. They further demonstrated that the basis of physical contact between HSCs and MSCs was dominated by the SDF-1/CXCR-4 and integrin axis. We also report the dominance of the SDF- $1 \alpha$ and integrin axis in our co-culture comprising PMSCs by a series of blocking experiments further underscoring the supremacy and specificity of such interactions. Thus, we believe that P-MSCs utilize similar cellular interactions as BM-MSCs for maintenance of primitive HSCs. The observed differences in the expanded cells could also be due to the differential survival of these subsets as MSC-HSCs co-cultures exhibited reduction in apoptosis of HSCs [29]. We observed striking differences in the survival of cells expanded on C-MSCs and on P-MSCs due to contrasting levels of Bcl-2 and Bax in the different subsets. Higher Bcl-2 levels were found in the primitive subset leading to the persistence of this population in P-MSCs co-cultures. Higher Bcl-2 levels are not only responsible for the survival of HSCs but several investigators have established its link with in vivo functionality of HSCs [30, 31]. We indeed found higher primary and secondary engraftment of HSCs expanded on P-MSCs than on CMSCs. The superior SRC potential of cells expanded on P-MSCs could be due to the higher migration towards SDF-1 $\alpha$ observed with these cells in vitro which might have resulted in better migration to $\mathrm{BM}$ in irradiated NOD/SCID mice. Second, being physically attached to the HSCs the P-MSCs infused along with the graft might be responsible for better engraftment. This observation is supported by findings of Hiwase et al. [32] who reported better engraftment in mice when transplanted with PMSCs along with HSCs. Probably the HSCs that are physically attached to P-MSCs are successfully delivered to the BM niche. Our observations between contact and noncontact cultures further strengthen this possibility. Moreover, P-MSCs were found to be more immature than C-MSCs as indicated by their higher proliferative potential and clonogenicity. This might be another reason for better engraftment of cells expanded on PMSCs as this conjecture is supported by the findings of Yim et al. [33].

To understand the molecular basis of the above observed differences we considered the stromal cell mediated Wnt/ $\beta$ catenin signaling and its effects on the HSCs. Activation of $\mathrm{Wnt} / \beta$-catenin signaling in stromal cells is shown to promote hematopoiesis by up-regulating the expression of the notch ligands (jagged-1, Dll-1) on stromal cells [34]. P-MSCs showed higher $\beta$-catenin levels which may have caused the higher expression of jagged 1 and Dll-1 on their surface resulting in a corresponding increase in the notch receptors and its responsive genes in the $\mathrm{CD}_{3}{ }^{+}$cells expanded on P-MSCs. We further tried to elevate the level of Wnt signaling in C-MSCs by lithium chloride treatment which resulted in a moderate improvement in the expansion of HSCs (data not shown). However, the contribution of the other signaling pathways is yet to be determined.

As a consequence of higher Wnt activity, MSC differentiation is skewed towards osteoblast over the other lineages [35]. Therefore, in an attempt to establish whether the observed differences in the two feeder cells might influence differentiation to osteoblasts, we checked the level of Runx-2 and found significantly higher expression in the P-MSCs. According to Chitteti et al. [36], primitive osteoblasts with high Runx-2 expression resulted in better promotion of HPC proliferation, additionally strengthening our observation of superior expansion of HSCs with pre-osteoblastic P-MSCs. Further, Runx-2 expressing preosteoblasts are predominantly found in the endosteum of the bones which is hypoxic in nature. To further confirm this, we checked the HIF-1 $\alpha$ levels; there was no difference in the HIF-1a stabilization in the two feeders when maintained under hypoxic conditions (data not shown). We indeed found that P-MSCs showed significantly higher nuclear stabilization of HIF-1 $\alpha$ under normoxic conditions, corroborating the reports by Palomaki et al. $[37,38]$. Collectively, our data strongly suggest that PMSCs partly provide conditions resembling the endosteal niche of the bone marrow. The similarity between the placenta and bone marrow could be explained by the fact 
that the placenta is a reservoir of HSCs during development [39], while the endosteum remains a reservoir of HSCs throughout life by maintaining steady state hematopoiesis establishing a solid link between bone marrow and placenta. However, we have not done direct comparisons of PMSCs with BM-MSCs in this respect as this was not the main objective of this manuscript. Nonetheless, we found that P-MSCs have a similar capacity to sustain ex vivo hematopoiesis as that of BM-MSCs. Thus, P-MSCs appear to be a non-invasive and clinically equivalent substitute for BM-MSCs to be used as feeders for expansion of HSCs.

\section{Conclusions}

In summary we report here that P-MSCs are more suitable as feeders than C-MSCs for expansion of UCBderived HSCs. Higher activity of Wnt/Notch signaling, normoxic HIF- $1 \alpha$ stabilization, pro-osteoblastic phenotype and HGF secretion along with a drastic reduction in apoptosis cumulatively add to the improved ability of HSCs expansion of P-MSCs. Current cell therapy protocols utilize C-MSCs as an alternative to BM-MSCs. However, our findings underscore the use of P-MSCs over C-MSCs for optimizing current protocols aimed at expanding HSCs. Further, since our HSC-MSC cocultures were grown in serum free conditions, they hold potential to be directly translated in the clinical settings.

\section{Additional files}

Additional file 1: Figure S1. BM-MSCs and P-MSCs had similar potentia for expansion of CD34+ cells. a BM-MSCs have fibroblastic morphology, can be differentiated into osteoblasts, adipocytes and chondrocytes. b BM-MSCs were shown to exhibit expression of CD44, CD73, CD105, and CD90 and found not to express CD34, CD45, CD14, CD19, CD11b, and andHLA-DR. c BM-MSCs had robust expansion of total nucleated cells, CD34 $4^{+} \mathrm{CD} 38^{-}$, and

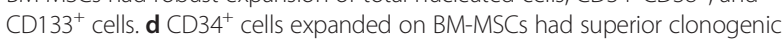
ability. Data are represented as mean \pm standard deviation from four different samples. ${ }^{*} p \leq 0.05,{ }^{* *} p \leq 0.01$, and ${ }^{* *} p \leq 0.001$. (TIFF 5033 kb)

Additional file 2: Figure S2. Cells expanded on P-MSCs gave rise to persistent engraftment in NOD/SCID mice. a Superior multi-lineage engraftment as denoted by donor-derived CD34 (stem cells), CD33 (myeloid cells), CD56 (NK cells), CD19 (B-cells), and CD3 (T-cells) after 12 weeks was detected in bone marrow of animals receiving cells from P-MSCs co-cultures as opposed to C-MSCs co-cultures $(n=10)$. b Mice infused with cells expanded on P-MSCs showed significantly higher numbers of donor-derived progenitors (CFU) after 12 weeks. c Multi-lineage engraftment in secondary recipient also showed superiority of the cells expanded on P-MSCs. $(n=9)$. $\mathbf{d}$ Secondary recipient similarly displayed a significantly higher number of human CFC in the mice receiving cells from primary recipient infused with cells from P-MSCs:CD34 ${ }^{+}$co-cultures. $(n=9)$. Data are represented as mean \pm standard deviation from ten different mice. ${ }^{*} \mathrm{p} \leq 0.05,{ }^{* *} \mathrm{p} \leq 0.01$, and ${ }^{* *} \mathrm{p} \leq 0.001$. (TIFF $2354 \mathrm{~kb}$ )

Additional file 3: Figure S3. Addition of apoptotic inhibitors in C-MSCs:CD34 ${ }^{+}$co-cultures improved expansion of UCB CD34 ${ }^{+}$cells. a Addition of pan caspase inhibitor (zVADfmk) and calpain-1 inhibitor (zLLYfmk) to the C-MSCs:CD34 ${ }^{+}$co-cultures significantly improved total nucleated cell yield in comparison to the control co-cultures. b Significant increase in the numbers of $\mathrm{CD} 34^{+} \mathrm{CD} 38^{-}$and $\mathrm{CD} 133^{+}$cells was detected in the co-cultures inclusive of zVADfmk and zLLYfmk as opposed to the ones without them. $\mathbf{c}, \mathbf{d}, \mathbf{e}$ Apoptotic inhibitors improved the in vitro functionality of the expanded cells as determined by CFU content (c), in vitro migration (d) and in vitro adhesion to fibronectin (e), respectively. Data are represented as mean \pm standard deviation from three different independent experimental sets. ${ }^{*} p \leq 0.05,{ }^{* *} p \leq 0.01$, and ${ }^{* * *} p \leq 0.001$. (TIFF 2676 kb)

Additional file 4: Figure S4. P-MSCs favors the expansion of CD34 cells in a contact dependent manner. a Significant reduction in total nucleated cells (TNC) was observed in non-contact cultures of P-MSCs while no effect was seen in non-contact cultures of C-MSCs. b Noncontact cultures of P-MSCs displayed reduction in the percentage of $\mathrm{CD}_{4}{ }^{+}$and $\mathrm{CD} 34^{+} \mathrm{CD} 38^{-}$cells as compared to contact cultures. c Reduction in the yield of TNC and CD34+ cells was due to an increase in the apoptosis in non-contact cultures as seen by a decrease in the annexinV ${ }^{-} \mathrm{Pl}^{-}$cells in non-contact cultures. $\mathbf{d}$ No change in the proliferation of $\mathrm{CD}_{3} 4^{+}$cells either singly blocked with CD49d (VLA-4), CD49e (VLA-5), and CXCR-4 or in combination with CD49d + CD49e and CD49d + CD49e + CXCR-4 was observed after co-culturing with C-MSCs for 96 hrs as assessed by MTT assay. e Drastic reduction was seen in the proliferation of $\mathrm{CD}_{3} 4^{+}$cells upon blocking with CD49d + CD49e, CXCR-4, and CD49d + CD49e + CXCR-4 and co-culturing with P-MSCs. $\mathbf{f}$ Clonogenecity of the cells expanded on C-MSCs remained unaltered even after blocking with anti-bodies. $\mathbf{g}$ Significant reduction was observed in the progenitor content after blocking the $\mathrm{CD}_{3}{ }^{+}$cells with the combination of antibodies and co-culture on P-MSCs. $\mathbf{h}$ Percentage of live population remains unchanged in the blocked set as opposed to the unblocked set in the co-cultures comprised of C-MSCs. $\mathbf{i}$ Viability of the expanded cells blocked with integrins and CXCR-4 reduced significantly in co-cultures with P-MSCs as feeders. Data are represented as mean \pm standard deviation from three different independent experimental sets. ${ }^{*} p \leq 0.05$, ${ }^{* *} \mathrm{p} \leq 0.01$, and ${ }^{* * *} \mathrm{p} \leq 0.001$. (TIFF $4452 \mathrm{~kb}$ )

\section{Abbreviations}

C-MSCs: Cord derived MSCs; HSCs: Hematopoietic stem cells; MSCs: Mesenchymal stem cells; P-MSCs: Placenta derived MSCs; P-MSCs: CD34+ co-cultures -CD34 cells expanded on P-MSCs; C-MSCs: CD34 ${ }^{+}$co-cultures-CD34 $4^{+}$cells expanded on C-MSCs.

\section{Competing interests}

The authors declare that they have no competing interests.

\section{Authors' contributions}

$\mathrm{LL}$ conceived and designed the experiments. DK performed the experiments. $D K, L L$, and VK analyzed the data. LL and VK contributed reagents/materials/ analysis tools. LL, VK, and DK wrote the paper. All authors read and approved the final manuscript.

\section{Acknowledgements}

We thank DRDO (LSRB), Government of India, New Delhi for funding the project. DK received a fellowship from University Grants Commission (UGC), New Delhi. We acknowledge Dr. Godbole, Dr. Prakash Daithankar and Dr. Bhosale for providing cord blood, cord and placenta samples. Special thanks to Dr. Mohan R. Wani for providing BM-MSCs. Thanks to Sonal Rangole and Nikhat Firdaus Q. Khan for technical help, core facilities of NCCS, such as FACS, Confocal and Animal House, and Dr. Jyoti Rao for proof reading of the manuscript.

\section{Endnote}

Some of this data was presented by Lalita Limaye at ISSCR-International Society for Stem Cell Research 11th Annual meeting held at Boston in June 2013.

Received: 3 March 2015 Revised: 15 July 2015

Accepted: 28 September 2015 Published online: 19 October 2015

\section{References}

1. Hofmeister CC, Zhang J, Knight KL, Le P, Stiff PJ. Ex vivo expansion of umbilical cord blood stem cells for transplantation: growing knowledge from the hematopoietic niche. Bone Marrow Transplant. 2007;39:11-23.

2. Ponce DM, Gonzales A, Lubin M, Castro-Malaspina H, Giralt S, Goldberg JD, et al. Graft-versus-host disease after double-unit cord blood transplantation has unique features and an association with engrafting unit-to-recipient HLA match. Biol Blood Marrow Transplant. 2013;6:904-11. 
3. Haspel RL, Ballen KK. Double cord blood transplants: filling a niche? Stem Cell Rev. 2006;2:81-6.

4. Flores-Guzman P, Fernandez-Sanchezv V, Mayani H. Concise review: ex vivo expansion of cord blood-derived hematopoietic stem and progenitor cells: basic principles, experimental approaches, and impact in regenerative medicine. Stem Cells Trans Med. 2013;2:830-8.

5. Kelly SS, Parmar S, De Lima M, Robinson S, Shpall E. Overcoming the barriers to umbilical cord blood transplantation. Cytotherapy. 2010;12:121-30.

6. Dahlberg A, Delaney C, Bernstein I. Ex vivo expansion of human hematopoietic stem and progenitor cells. Blood. 2011;117:6083-90.

7. Mayani H, Guilbert L, Janowska-Wieczorek A. Biology of the hemopoietic microenvironment. Eur J Haematol. 1992;49:225-33.

8. Robinson SN, Ng J, Niu T, Yang H, McMannis JD, Karandish S, et al. Superior ex vivo cord blood expansion following co-culture with bone marrowderived mesenchymal stem cells. Bone Marrow Transplant. 2006;37:359-66.

9. Salati S, Lisignoli G, Manferdini C, Pennucci V, Zini R, Bianchi E, et al. Co-culture of hematopoietic stem/progenitor cells with human osteoblasts favours mono/macrophage differentiation at the expense of the erythroid lineage. PLoS One. 2013;8:e53496. doi:10.1371/journal.pone.0053496.

10. Rosler E, Brandt J, Chute JV, Hoffman R. Co cultivation of umbilical cord blood cells with endothelial cells leads to extensive amplification of competent CD34 ${ }^{+}$CD38 ${ }^{-}$cells. Exp Hematol. 2000;28:841-52.

11. Magnusson M, Sierra MI, Sasidharan R, Prashad SL, Romero M, Saarikoski P, et al. Expansion on stromal cells preserves the undifferentiated state of human hematopoietic stem cells despite compromised reconstitution ability. PLoS One. 2013;8:e53912. doi:10.1371/journal.pone.0053912.

12. Klein C, Strobel J, Zingsem J, Richter R, Goecke T, Beckmann M, et al. Ex vivo expansion of hematopoietic stem- and progenitor cells from cord blood in coculture with mesenchymal stroma cells from amnion, chorion, Wharton's jelly, amniotic fluid, cord blood, and bone marrow. Tissue Eng A. 2013;19:2577-85.

13. Hass R, Kasper C, Böhm S, Jacobs R. Different populations and sources of human mesenchymal stem cells (MSC): a comparison of adult and neonatal tissue-derived MSC. Cell Commun Signal. 2011;9:12

14. Wegmeyer H, Bröske AM, Leddin M, Kuentzer K, Nisslbeck AK, Hupfeld J, et al. Mesenchymal stromal cell characteristics vary depending on their origin. Stem Cells Dev. 2013;19:2606-18.

15. Talwadekar M, Kadekar D, Rangole S, Khan NF, Kale V, Limaye L. Cultivation and cryopreservation of cord tissue MSCs with cord blood AB plasma. Biomed Res J. 2014:1:126-36.

16. Dominici M, Le Blanc K, Mueller I, Slaper-Cortenbach I, Marini F, Krause D, et al. Minimal criteria for defining multipotent mesenchymal stromal cells. The International Society for Cellular Therapy position statement. Cytotherapy. 2006:8:315-7.

17. Sangeetha VM, Kale VP, Limaye LS. Expansion of cord blood CD34 $4^{+}$cells in presence of zVADfmk and zLLYfmk improved their in vitro functionality and in vivo engraftment in NOD/SCID mouse. PLoS One. 2010;5:e12221. doi:10.1371/journal.pone.0012221

18. Yi Z, Li C, Jiang X, Zhang S, Wu Y, Liu B, et al. Human placenta-derived mesenchymal progenitor cells support culture expansion of long-term culture-initiating cells from cord blood CD34 ${ }^{+}$cells. Exp Hematol. 2004;32:657-64.

19. Bakhshi T, Zabriskie R, Bodie S, Kidd S, Ramin S, Paganessi L, et al. Mesenchymal stem cells from the Wharton's jelly of umbilical cord segments provide stromal support for the maintenance of cord blood hematopoietic stem cells during long-term ex vivo culture. Transfusion. 2008:48:2638-47.

20. Celebi B, Mantovani D, Pineault N. Irradiated mesenchymal stem cells improve the ex vivo expansion of hematopoietic rogenitors by partly mimicking the bone marrow endosteal environment. J Immunol Methods. 2011;370:93-103

21. Walenda T, Bork S, Horn P, Wein F, Saffrich R, Diehlmann A, et al. Co-culture with mesenchymal stromal cells increases proliferation and maintenance of haematopoietic progenitor cells. J Cell Mol. 2010;14:337-50.

22. Mayani H, Little MT, Dragowska W, Thornbury G, Lansdorp PM. Differential effects of the hematopoietic inhibitors MIP-1 alpha, TGF-beta, and TNFalpha on cytokine-induced proliferation of subpopulations of CD34+ cells purified from cord blood and fetal liver. Exp Hematol. 1995;5:422-7.

23. Hwang JH, Shim SS, Seok OS, Lee HY, Woo SK, Kim BH, et al. Comparison of cytokine expression in mesenchymal stem cells from human placenta, cord blood, and bone marrow. J Korean Med Sci. 2009;24:547-54.
24. Wagner W, Roderburg C, Wein F, Diehlmann A, Frankhauser M, Schubert R, et al. Molecular and secretory profiles of human mesenchymal stromal cells and their abilities to maintain primitive hematopoietic progenitors. Stem Cells. 2007;25:2638-47.

25. Fong CY, Gauthaman K, Cheyyatraivendran S, Lin HD, Biswas A, Bongso A. Human umbilical cord Wharton's jelly stem cells and its conditioned medium support hematopoietic stem cell expansion ex vivo. J Cell Biochem. 2012;113:658-68.

26. Da Silva C, Gonçalves R, dos Santos F, Andrade P, Almeida-Porada G, Cabral J. Dynamic cell-cell interactions between cord blood haematopoietic progenitors and the cellular niche are essential for the expansion of CD34+, CD34 + CD38- and early lymphoid CD7+ cells. J Tissue Eng Regen Med. 2010;: :149-58

27. Jung Y, Wang J, Havens A, Sun Y, Wang J, Jin T, et al. Cell-to- cell contact is critical for the survival of hematopoietic progenitor cells on osteoblasts. Cytokine. 2005;32:155-62.

28. Jing $\mathrm{D}$, Fonseca AV, Alakel N, Fierro FA, Muller K, Bornhauser M, et al. Hematopoietic stem cells in co-culture with mesenchymal stromal cells - modeling the niche compartments in vitro. Haematologica. 2010;95:542-50

29. Mehrasa R, Vaziri H, Oodi A, Khorshidfar M, Nikogoftar M, Golpour M, et al. Mesenchymal stem cells as a feeder layer can prevent apoptosis of expanded hematopoietic stem cells derived from cord blood. Int J Mol Cell Med. 2014;3:1-10

30. Domen J, Cheshier S, Weissman I. Role of apoptosis in hematopoietic stem cell homeostasis. J Exp Med. 2000;191:253-63.

31. Orelio C, Dzierzak E. BCl-2 expression and apoptosis in the regulation of hematopoietic stem cells. Leuk Lymphoma. 2007;48:16-24.

32. Hiwase S, Dyson P, To L, Lewis I. Cotransplantation of placental mesenchymal stromal cells enhances single and double cord blood engraftment in nonobese diabetic/severe combined immune deficient mice. Stem Cells. 2009;27:2293-300.

33. Yim Y, Noh Y, Kim D, Lee M, Cheuh H, Lee S, et al. Correlation between the immature characteristics of umbilical cord blood-derived mesenchymal stem cells and engraftment of hematopoietic stem cells in NOD/SCID mice. Transplant Proc. 2010;42:2753-8.

34. Kim J, Kang Y, Park G, Kim M, Park Y, Kim H, et al. Identification of a stroma-mediated Wnt/b-catenin signal promoting self-renewal of hematopoietic stem cells in the stem cell niche. Stem Cells. 2009;27:1318-29.

35. James A. Review of signaling pathways governing MSC osteogenic and adipogenic differentiation. Scientifica. 2013;2013:684736-17.

36. Chitteti B, Cheng Y, Streicher D, Rodriguez S, Carlesso N, Srour E, et al. Osteoblast lineage cells expressing high levels of Runx2 enhance hematopoietic progenitor cell proliferation and function. J Cell Biochem. 2010;111:284-94

37. Palomäki S, Pietilä M, Laitinen S, Pesälä J, Sormunen R, Lehenkari P, et al. HIF-1a is upregulated in human mesenchymal stem cells. Stem Cells. 2013;31:1902-9.

38. Guarnerio J, Coltella N, Ala U, Tonon G, Pandolfi P, Bernardi R. Bone marrow endosteal mesenchymal progenitors depend on HIF factors for maintenance and regulation of hematopoiesis. Stem Cell Reports. 2014;2:794-809.

39. Robin C, Bollerot K, Mendes S, Haak E, Crisan M, Cerisoli F, et al. Human placenta is a potent hematopoietic niche containing hematopoietic stem and progenitor cells throughout development. Cell Stem Cells. 2009:5:385-95. 